\title{
BUSEMANN'S INTERSECTION INEQUALITY IN HYPERBOLIC AND SPHERICAL SPACES
}

\author{
SUSANNA DANN, JAEGIL KIM, AND VLADYSLAV YASKIN
}

\begin{abstract}
Busemann's intersection inequality asserts that the only maximizers of the integral $\int_{S^{n-1}}\left|K \cap \xi^{\perp}\right|^{n} d \xi$ among all convex bodies of a fixed volume in $\mathbb{R}^{n}$ are centered ellipsoids. We study this question in the hyperbolic and spherical spaces, as well as general measure spaces.
\end{abstract}

\section{INTRODUCTION}

Let $K$ be a convex body in $\mathbb{R}^{n}$ that contains the origin in its interior. The following is known as Busemann's intersection inequality:

$$
\int_{S^{n-1}}\left|K \cap \xi^{\perp}\right|^{n} d \xi \leq c_{n}|K|^{n-1},
$$

with equality if and only if $K$ is a centered ellipsoid; see [1]. Here, $c_{n}=$ $n \kappa_{n-1}^{n} / \kappa_{n}^{n-2}$, where $\kappa_{n}$ is the volume of the unit Euclidean ball $B^{n}$ in $\mathbb{R}^{n}$, and $|A|$ stands for the volume (in the appropriate dimension) of a set $A$.

In fact, the inequality (1) is true for a larger class of sets, in particular, star bodies; see [3, p.373]. In a slightly different form, (1) can be stated as follows. Centered ellipsoids in $\mathbb{R}^{n}$ are the only maximizers of the quantity

$$
\int_{S^{n-1}}\left|K \cap \xi^{\perp}\right|^{n} d \xi
$$

in the class of star bodies of a fixed volume. In this paper we study this question in the hyperbolic space $\mathbb{H}^{n}$ and the sphere $\mathbb{S}^{n}$ (or, more precisely, a hemisphere $\mathbb{S}_{+}^{n}$, as explained in the next section). We show that in $\mathbb{H}^{n}$ centered balls are the unique maximizers of (2) in the class of star bodies of a fixed volume. On the sphere the situation is different. In $\mathbb{S}_{+}^{2}$ centered balls are in fact the unique minimizers (in the class of origin-symmetric star bodies). The maximizers of (2) in the class of origin-symmetric star bodies in $\mathbb{S}_{+}^{2}$ are cones (see Section 2 for the definition). The maximizers of $(2)$ in the class of origin-symmetric convex bodies in $\mathbb{S}_{+}^{2}$ are lunes. It is surprising that in $\mathbb{S}_{+}^{n}$ with $n \geq 3$ centered balls are neither maximizers nor minimizers, even in the class of origin-symmetric convex bodies. We also obtain an

Date: September 10, 2018.

2010 Mathematics Subject Classification. Primary: 52A55, 52A20, 52A38, 52A40.

Key words and phrases. convex bodies, sections, hyperbolic and spherical spaces, Radon transform, spherical harmonics.

The second and third named authors are supported in part by NSERC. 
optimal lower bound for (2) in the class of star bodies in $\mathbb{S}_{+}^{n}, n \geq 3$, of a given volume and describe the equality cases. Finally, we prove a version of Busemann's intersection inequality (together with the equality cases) for general measures on $\mathbb{R}^{n}$ and $\mathbb{H}^{n}$. An important special case is that of the Gaussian measure on $\mathbb{R}^{n}$.

For the history of Busemann's inequality, its applications, and recent developments the reader is referred to [2, [3, 4], [5], 7].

It is interesting to note that, in the context of the Busemann-Petty problem, the sphere and the Euclidean space are similar in the sense that the positive answer holds in the same dimensions, while the hyperbolic space exhibits a different behaviour; see [8]. For Busemann's intersection inequality, the hyperbolic space is similar to the Euclidean space, while the sphere is not.

\section{Preliminaries}

We will start with some basic facts about the hyperbolic space $\mathbb{H}^{n}$ and the sphere $\mathbb{S}^{n}$. In $\mathbb{H}^{n}$ any two points are connected by a unique geodesic line. Thus we say that a set $A$ in $\mathbb{H}^{n}$ is convex if for any two points that belong to $A$, the geodesic segment that connects these two points also belongs to $A$. On the sphere $\mathbb{S}^{n}$ the uniqueness property of geodesics does not hold. To overcome this obstacle, one can consider an open hemisphere, where geodesic convexity is well-defined. We will however need a larger class of convex sets. Let $\mathbb{S}_{+}^{n}$ be the closed upper hemisphere of the unit sphere in $\mathbb{R}^{n+1}$. A set $A \subset \mathbb{S}_{+}^{n}$ is said to be convex if $A$ is obtained as the intersection of the hemisphere $\mathbb{S}_{+}^{n} \subset \mathbb{R}^{n+1}$ and a convex cone in $\mathbb{R}^{n+1}$ with vertex at the origin. One can see that the class of convex sets contains all geodesically convex sets in the interior of $\mathbb{S}_{+}^{n}$, as well as certain sets that have common points with the relative boundary of $\mathbb{S}_{+}^{n}$.

To treat both spaces simultaneously, we will denote them by $\mathbb{M}_{\delta}^{n}$, where $\delta=-1$ corresponds to $\mathbb{H}^{n}$ and $\delta=1$ to $\mathbb{S}_{+}^{n}$. The definition of star bodies in $\mathbb{M}_{\delta}^{n}$ is similar to that in the Euclidean space. More precisely, first fix an origin $o$ in $\mathbb{M}_{\delta}^{n}$ and denote by $T_{o} \mathbb{M}_{\delta}^{n}$ the tangent space to $\mathbb{M}_{\delta}^{n}$ at $o$. In the case of $\mathbb{S}_{+}^{n}$, the origin is the center of the hemisphere. We say that a subset $K$ of $\mathbb{M}_{\delta}^{n}$ is star-shaped (with respect to $o$ ), if every geodesic passing through $o$ intersects $K$ in a connected segment containing $o$. Consider the unit sphere $S^{n-1}$ in $T_{o} \mathbb{M}_{\delta}^{n}$, i.e. the set of vectors in $T_{o} \mathbb{M}_{\delta}^{n}$ that have length one with respect to the metric of $\mathbb{M}_{\delta}^{n}$. For each $\xi \in S^{n-1} \subset T_{o} \mathbb{M}_{\delta}^{n}$, consider the geodesic ray $\gamma$ emanating from $o$ with tangent vector $\xi$. The radial function of $K$ in the direction of $\xi$ is defined by

$$
\rho_{K}(\xi)=\sup _{x \in \gamma \cap K} \mathrm{~d}(x, o),
$$

where $\mathrm{d}$ is the metric in $\mathbb{M}_{\delta}^{n}$. 
We say that $K$ is a star body in $\mathbb{M}_{\delta}^{n}$ if $K$ is compact, star-shaped with respect to $o$, and its radial function is positive and continuous. A starshaped set $K$ is origin-symmetric if $\rho_{K}(\xi)=\rho_{K}(-\xi)$ for all $\xi \in S^{n-1}$. For each $\xi \in S^{n-1} \subset T_{o} \mathbb{M}_{\delta}^{n}$, we denote by $\xi^{\perp}$ the (unique) totally geodesic submanifold of $\mathbb{M}_{\delta}^{n}$ passing through $o$, whose normal vector at $o$ is $\xi$. As in the Euclidean space, we will often refer to $\xi^{\perp}$ as a hyperplane.

The space $\mathbb{M}_{\delta}^{n}$ for both $\delta= \pm 1$ can be identified with the unit ball $B^{n} \subset$ $\mathbb{R}^{n}$ (open when $\delta=-1$ and closed when $\delta=1$ ) equipped with the metric

$$
d s^{2}=4 \frac{d x_{1}^{2}+\cdots+d x_{n}^{2}}{\left(1+\delta\left(x_{1}^{2}+\cdots+x_{n}^{2}\right)\right)^{2}} .
$$

In this model star bodies in $\mathbb{M}_{\delta}^{n}$ correspond to Euclidean star bodies in the ball $B^{n}$. Thinking of $K$ as a body in $B^{n} \subset \mathbb{R}^{n}$, we can use standard Euclidean concepts. The Minkowski functional of $K \subset \mathbb{R}^{n}$ is defined by

$$
\|x\|_{K}=\min \{a \geq 0: x \in a K\}, \quad x \in \mathbb{R}^{n} .
$$

In metric (3) the volume of $K$ equals

$$
\operatorname{vol}(K)=2^{n} \int_{S^{n-1}} \int_{0}^{\|\theta\|_{K}^{-1}} \frac{r^{n-1} d r}{\left(1+\delta r^{2}\right)^{n}} d \theta
$$

where, once again, $\|\theta\|_{K}$ is well-defined, since $K$ is a body in $B^{d} \subset \mathbb{R}^{n}$.

The volume of the section of $K$ by the hyperplane $\xi^{\perp}$ is given by

$$
\operatorname{vol}\left(K \cap \xi^{\perp}\right)=2^{n-1} \int_{S^{n-1} \cap \xi^{\perp}} \int_{0}^{\|\theta\|_{K}^{-1}} \frac{r^{n-2} d r}{\left(1+\delta r^{2}\right)^{n-1}} d \theta .
$$

When a body $K$ lies in the Euclidean space, by $|K|$ and $\left|K \cap \xi^{\perp}\right|$ we denote its volume and the volume of its sections with respect to the Euclidean metric.

We will also use another coordinate system for $\mathbb{M}_{\delta}^{n}$, in which

$$
\operatorname{vol}(K)=\int_{S^{n-1}} \int_{0}^{\rho_{K}(\theta)} s_{\delta}^{n-1}(r) d r d \theta
$$

and

$$
\operatorname{vol}\left(K \cap \xi^{\perp}\right)=\int_{S^{n-1} \cap \xi^{\perp}} \int_{0}^{\rho_{K}(\theta)} s_{\delta}^{n-2}(r) d r d \theta,
$$

where $s_{1}(r)=\sin r$ and $s_{-1}(r)=\sinh r$.

Below we introduce some concepts specific to the spherical case. We say that a star-shaped subset $K$ of $\mathbb{S}_{+}^{n}$ is a spherical cone (or simply a cone in $\mathbb{S}_{+}^{n}$ ) if the radial function of $K$ is equal to $\pi / 2$ on its support. In this case, the support of the radial function, which is a subset of $S^{n-1}$, is called the base of the cone.

Two geodesic lines in $\mathbb{S}_{+}^{2}$ are said to be parallel to each other if they never meet in the interior of $\mathbb{S}_{+}^{2}$, i.e., if they meet only on the boundary of $\mathbb{S}_{+}^{2}$. A 
region in $\mathbb{S}_{+}^{2}$ is called a lune if its boundary consists of two parallel geodesic lines.

We will use some tools of harmonic analysis, in particular, the spherical Radon transform and spherical harmonics. Recall that for a function $f \in$ $C\left(S^{n-1}\right)$ its spherical Radon transform $R f$ is the function on $S^{n-1}$ given by

$$
R f(\xi)=\int_{S^{n-1} \cap \xi^{\perp}} f(\theta) d \theta, \quad \xi \in S^{n-1} .
$$

On the spherical harmonics of a fixed degree the operator $R: C\left(S^{n-1}\right) \rightarrow$ $C\left(S^{n-1}\right)$ acts as a multiple of the identity. Namely, if $H_{k}$ is a spherical harmonic of an even degree $k$, then

$$
R H_{k}=\lambda_{k} H_{k},
$$

where

$$
\lambda_{k}=\frac{(-1)^{k / 2} 2 \pi^{(n-2) / 2} \Gamma((k+1) / 2)}{\Gamma((n+k-1) / 2)} ;
$$

cf., for example, [6, p.103]. For odd $k$ all the multipliers are equal to zero.

Observe that $\left|\lambda_{k}\right|$, for even $k$, form a strictly decreasing sequence tending to zero as $k \rightarrow \infty$. Since $\lambda_{0}=\left|S^{n-2}\right|$, the latter implies that

$$
\|R f\|_{L^{2}\left(S^{n-1}\right)} \leq\left|S^{n-2}\right|\|f\|_{L^{2}\left(S^{n-1}\right)} .
$$

We will often use the following relation:

$$
\int_{S^{n-1}} \int_{S^{n-1} \cap \xi^{\perp}} g(\theta) d \theta d \xi=\left|S^{n-2}\right| \int_{S^{n-1}} g(\xi) d \xi .
$$

\section{BusemanN'S INTERSECTION INEQUALITy IN THE HyPERBoliC SPACE}

Before we state our results, let us introduce the following functions. For $n \geq 1$, let the function $F_{n}:[0,1) \rightarrow[0, \infty)$ be defined by

$$
F_{n}(t)=\int_{0}^{t} \frac{r^{n-1}}{\left(1-r^{2}\right)^{n}} d r .
$$

If $n \geq 2$, consider the function

$$
G(t)=\left[F_{n-1}\left(F_{n}^{-1}(t)\right)\right]^{n /(n-1)}, \quad t \in[0, \infty) .
$$

Lemma 3.1. The function $G$ is concave.

Proof. Since

$$
G\left(F_{n}(t)\right)=\left[F_{n-1}(t)\right]^{n /(n-1)}, \quad t \in[0,1),
$$

we have

$$
G^{\prime}\left(F_{n}(t)\right) \frac{t^{n-1}}{\left(1-t^{2}\right)^{n}}=\frac{n}{n-1}\left[F_{n-1}(t)\right]^{1 /(n-1)} \frac{t^{n-2}}{\left(1-t^{2}\right)^{n-1}} .
$$

Thus, we obtain

$$
G^{\prime}\left(F_{n}(t)\right)=\frac{n}{n-1}\left[F_{n-1}(t)\right]^{1 /(n-1)}\left(\frac{1}{t}-t\right) .
$$


Differentiating one more time, we get

$$
\begin{aligned}
G^{\prime \prime}\left(F_{n}(t)\right) \frac{t^{n-1}}{\left(1-t^{2}\right)^{n}}=\frac{n}{(n-1)^{2}} & {\left[F_{n-1}(t)\right]^{(-n+2) /(n-1)} \frac{t^{n-3}}{\left(1-t^{2}\right)^{n-2}} } \\
& +\frac{n}{n-1}\left[F_{n-1}(t)\right]^{1 /(n-1)}\left(-\frac{1}{t^{2}}-1\right) .
\end{aligned}
$$

To show that $G^{\prime \prime}<0$, we need to prove the inequality

$$
(n-1) F_{n-1}(t) \geq \frac{t^{n-1}}{\left(1-t^{2}\right)^{n-2}\left(1+t^{2}\right)}, \quad t \in[0,1) .
$$

The functions on both sides of the inequality vanish at $t=0$. Thus it is enough to show that the same inequality holds for their derivatives, i.e., we need to show that

$$
\begin{aligned}
& (n-1) \frac{t^{n-2}}{\left(1-t^{2}\right)^{n-1}} \geq(n-1) t^{n-2}\left(1-t^{2}\right)^{-n+2}\left(1+t^{2}\right)^{-1} \\
& -2 t^{n}(-n+2)\left(1-t^{2}\right)^{-n+1}\left(1+t^{2}\right)^{-1}-2 t^{n}\left(1-t^{2}\right)^{-n+2}\left(1+t^{2}\right)^{-2},
\end{aligned}
$$

or equivalently,

$$
\begin{aligned}
(n-1)\left(1+t^{2}\right)^{2} \geq & (n-1)\left(1-t^{2}\right)\left(1+t^{2}\right) \\
& -2 t^{2}(-n+2)\left(1+t^{2}\right)-2 t^{2}\left(1-t^{2}\right) \\
= & (n-1)+(2 n-6) t^{2}+(n-1) t^{4} .
\end{aligned}
$$

This is true, since

$(n-1)+(2 n-6) t^{2}+(n-1) t^{4} \leq(n-1)+2(n-1) t^{2}+(n-1) t^{4}=(n-1)\left(1+t^{2}\right)^{2}$.

Let us introduce a new function

$$
H(t)=\left[G\left(\frac{t}{2^{n}\left|S^{n-1}\right|}\right)\right]^{n-1}, \quad t \geq 0 .
$$

Theorem 3.2. Let $K$ be a star body in $\mathbb{H}^{n}$ for $n \geq 2$. Then

$$
\int_{S^{n-1}} \operatorname{vol}\left(K \cap \xi^{\perp}\right)^{n} d \xi \leq C_{n} H(\operatorname{vol}(K)),
$$

with equality if and only if $K$ is a ball centered at the origin. Here,

$$
C_{n}=\left|S^{n-1}\right|^{n-1} n^{2} 2^{n(n-1)}\left(1-\frac{1}{n}\right)^{n} \frac{\kappa_{n-1}^{n}}{\kappa_{n}^{n-2}} .
$$

Proof. We will work in the Poincaré model of $\mathbb{H}^{n}$ in the Euclidean ball $B^{n}$, so

$$
\int_{S^{n-1}} \operatorname{vol}\left(K \cap \xi^{\perp}\right)^{n} d \xi=\int_{S^{n-1}}\left(\int_{S^{n-1} \cap \xi^{\perp}} \int_{0}^{\|\theta\|_{K}^{-1}} \frac{2^{n-1} r^{n-2}}{\left(1-r^{2}\right)^{n-1}} d r d \theta\right)^{n} d \xi
$$


Let $L$ be a star body in $\mathbb{R}^{n}$ whose Minkowski functional is given by

$$
\|\theta\|_{L}^{-1}=\left(\int_{0}^{\|\theta\|_{K}^{-1}} \frac{2^{n-1} r^{n-2}}{\left(1-r^{2}\right)^{n-1}} d r\right)^{1 /(n-1)} .
$$

Then

$$
\begin{aligned}
\int_{S^{n-1}} \operatorname{vol}\left(K \cap \xi^{\perp}\right)^{n} d \xi & =\int_{S^{n-1}}\left(\int_{S^{n-1} \cap \xi^{\perp}}\|\theta\|_{L}^{-n+1} d \theta\right)^{n} d \xi \\
& =(n-1)^{n} \int_{S^{n-1}}\left|L \cap \xi^{\perp}\right|^{n} d \xi
\end{aligned}
$$

We can now use Busemann's intersection inequality in $\mathbb{R}^{n}$.

$$
\begin{aligned}
& \leq n(n-1)^{n} \frac{\kappa_{n-1}^{n}}{\kappa_{n}^{n-2}}|L|^{n-1}=n(n-1)^{n} \frac{\kappa_{n-1}^{n}}{\kappa_{n}^{n-2}}\left(\frac{1}{n} \int_{S^{n-1}}\|\theta\|_{L}^{-n} d \theta\right)^{n-1} \\
& =n^{2} 2^{n(n-1)}\left(1-\frac{1}{n}\right)^{n} \frac{\kappa_{n-1}^{n}}{\kappa_{n}^{n-2}}\left[\int_{S^{n-1}}\left(\int_{0}^{\|\theta\|_{K}^{-1}} \frac{r^{n-2} d r}{\left(1-r^{2}\right)^{n-1}}\right)^{\frac{n}{n-1}} d \theta\right]^{n-1},
\end{aligned}
$$

where the equality case in the above inequality holds if and only if $L$ is a centered ellipsoid.

$$
\begin{aligned}
& =n^{2} 2^{n(n-1)}\left(1-\frac{1}{n}\right)^{n} \frac{\kappa_{n-1}^{n}}{\kappa_{n}^{n-2}}\left[\int_{S^{n-1}}\left(F_{n-1}\left(\|\theta\|_{K}^{-1}\right)\right)^{n /(n-1)} d \theta\right]^{n-1} \\
& =n^{2} 2^{n(n-1)}\left(1-\frac{1}{n}\right)^{n} \frac{\kappa_{n-1}^{n}}{\kappa_{n}^{n-2}}\left[\int_{S^{n-1}} G\left(F_{n}\left(\|\theta\|_{K}^{-1}\right)\right) d \theta\right]^{n-1} \\
& =\left|S^{n-1}\right|^{n-1} n^{2} 2^{n(n-1)}\left(1-\frac{1}{n}\right)^{n} \frac{\kappa_{n-1}^{n}}{\kappa_{n}^{n-2}}\left[\int_{S^{n-1}} G\left(F_{n}\left(\|\theta\|_{K}^{-1}\right)\right) \frac{d \theta}{\left|S^{n-1}\right|}\right]^{n-1} .
\end{aligned}
$$

By Jensen's inequality,

$$
\leq\left|S^{n-1}\right|^{n-1} n^{2} 2^{n(n-1)}\left(1-\frac{1}{n}\right)^{n} \frac{\kappa_{n-1}^{n}}{\kappa_{n}^{n-2}}\left[G\left(\int_{S^{n-1}} F_{n}\left(\|\theta\|_{K}^{-1}\right) \frac{d \theta}{\left|S^{n-1}\right|}\right)\right]^{n-1} .
$$

Since $G$ is not affine (the proof of Lemma 3.1 shows that its second derivative is strictly negative), the equality holds if and only if $F_{n}\left(\|\theta\|_{K}^{-1}\right)$ is constant on the sphere, i.e. $\|\theta\|_{K}^{-1}$ is constant on the sphere, meaning that $K$ is a ball centered at the origin.

Finally,

$$
\begin{aligned}
\int_{S^{n-1}} \operatorname{vol}\left(K \cap \xi^{\perp}\right)^{n} d \xi & \leq C_{n}\left[G\left(\int_{S^{n-1}} \int_{0}^{\|\theta\|_{K}^{-1}} \frac{r^{n-1} d r}{\left(1-r^{2}\right)^{n}} \frac{d \theta}{\left|S^{n-1}\right|}\right)\right]^{n-1} \\
& =C_{n}\left[G\left(\frac{\operatorname{vol}(K)}{2^{n}\left|S^{n-1}\right|}\right)\right]^{n-1}=C_{n} H(\operatorname{vol}(K))
\end{aligned}
$$




\section{Busemann's intersection inequality in the SPHERICAL SPACE: FIRST GLANCE}

The approach used in the previous section does not work for the spherical space, because the analogue of the function $G$ in the spherical setting fails to possess the needed convexity properties. However, it is possible to obtain weaker estimates.

Let us introduce a function $F$ by the formula

$$
F\left(\int_{0}^{t} \frac{r^{n-1}}{\left(1+r^{2}\right)^{n}} d r\right)=\int_{0}^{t} \frac{r^{n-2}}{\left(1+r^{2}\right)^{n-1}} d r, \quad t \geq 0 .
$$

Proposition 4.1. Let $K$ be a star body in $\mathbb{S}_{+}^{n}$. Then

$$
\int_{S^{n-1}} \operatorname{vol}\left(K \cap \xi^{\perp}\right) d \xi \leq 2^{n-1}\left|S^{n-1}\right|\left|S^{n-2}\right| F\left(\frac{1}{\left|S^{n-1}\right|} \operatorname{vol}(K)\right),
$$

with equality if and only if $K$ is a ball centered at the origin.

Proof. It is not hard to check that $F$ is concave. Thus,

$$
\begin{aligned}
\int_{S^{n-1}} & \operatorname{vol}\left(K \cap \xi^{\perp}\right) d \xi \\
& =2^{n-1} \int_{S^{n-1}} \int_{S^{n-1} \cap \xi^{\perp}} \int_{0}^{\|\theta\|_{K}^{-1}} \frac{r^{n-2}}{\left(1+r^{2}\right)^{n-1}} d r d \theta d \xi \\
& =2^{n-1}\left|S^{n-2}\right| \int_{S^{n-1}} \int_{0}^{\|\theta\|_{K}^{-1}} \frac{r^{n-2}}{\left(1+r^{2}\right)^{n-1}} d r d \theta \\
& =2^{n-1}\left|S^{n-2}\right| \int_{S^{n-1}} F\left(\int_{0}^{\|\theta\|_{K}^{-1}} \frac{r^{n-1}}{\left(1+r^{2}\right)^{n}} d r\right) d \theta \\
& \leq 2^{n-1}\left|S^{n-1}\right|\left|S^{n-2}\right| F\left(\frac{1}{\left|S^{n-1}\right|} \int_{S^{n-1}}^{\|\theta\|_{K}^{-1}} \frac{r^{n-1}}{\left(1+r^{2}\right)^{n}} d r d \theta\right) \\
& =2^{n-1}\left|S^{n-1}\right|\left|S^{n-2}\right| F\left(\frac{1}{\left|S^{n-1}\right|} \operatorname{vol}(K)\right) .
\end{aligned}
$$

Next we will prove a version of Busemann's intersection inequality in $\mathbb{S}_{+}^{n}$, which is, however, not optimal.

Proposition 4.2. Let $K$ be a star body in $\mathbb{S}_{+}^{n}$. Then

$$
\int_{S^{n-1}} \operatorname{vol}\left(K \cap \xi^{\perp}\right)^{n} d \xi<\frac{2^{n-1} n \kappa_{n-1}^{n}}{\kappa_{n}^{n-2}}[\operatorname{vol}(K)]^{n-1} .
$$

Proof. It was shown in [2] that if $f$ is a non-negative bounded integrable function on $\mathbb{R}^{n}$, then

$$
\int_{S^{n-1}} \frac{\left(\int_{\xi^{\perp}} f(x) d x\right)^{n}}{\left\|\left.f\right|_{\xi^{\perp}}\right\|_{\infty}} d \xi \leq \frac{n \kappa_{n-1}^{n}}{\kappa_{n}^{n-2}}\left(\int_{\mathbb{R}^{n}} f(x) d x\right)^{n-1} .
$$


Now regard $K$ as a star body in $B^{n}$ and use the latter formula with

$$
f(x)=\chi_{K}(x) \frac{2^{n-1}}{\left(1+|x|^{2}\right)^{n-1}} .
$$

Since

$$
\left\|\left.f\right|_{\xi^{\perp}}\right\|_{\infty}=2^{n-1}
$$

for every $\xi \in S^{n-1}$, we get

$$
\frac{1}{2^{n-1}} \int_{S^{n-1}}\left[\int_{K \cap \xi^{\perp}} \frac{2^{n-1} d x}{\left(1+|x|^{2}\right)^{n-1}}\right]^{n} d \xi \leq \frac{n \kappa_{n-1}^{n}}{\kappa_{n}^{n-2}}\left[\int_{K} \frac{2^{n-1} d x}{\left(1+|x|^{2}\right)^{n-1}}\right]^{n-1},
$$

that is

$$
\int_{S^{n-1}}\left[\operatorname{vol}\left(K \cap \xi^{\perp}\right)\right]^{n} d \xi \leq \frac{2^{n-1} n \kappa_{n-1}^{n}}{\kappa_{n}^{n-2}}\left[\int_{K} \frac{2^{n-1} d x}{\left(1+|x|^{2}\right)^{n-1}}\right]^{n-1} .
$$

Let us now estimate the right-hand side.

$$
\int_{K} \frac{2^{n-1}}{\left(1+|x|^{2}\right)^{n-1}} d x \leq \int_{K} \frac{2^{n}}{\left(1+|x|^{2}\right)^{n}} d x=\operatorname{vol}(K) .
$$

Finally,

$$
\int_{S^{n-1}} \operatorname{vol}\left(K \cap \xi^{\perp}\right)^{n} d \xi \leq \frac{2^{n-1} n \kappa_{n-1}^{n}}{\kappa_{n}^{n-2}}(\operatorname{vol}(K))^{n-1}
$$

\section{Busemann's intersection InEQUALity IN $\mathbb{S}_{+}^{2}$}

From the proof of Theorem 4.2 it is clear that the inequality obtained therein is not optimal. Our goal is to establish an optimal inequality and we will start with the case of $\mathbb{S}_{+}^{2}$. Here we will obtain sharp estimates for the minimum and maximum of $\int_{S^{1}} \operatorname{vol}\left(K \cap \xi^{\perp}\right)^{2} d \xi$ in the class of originsymmetric star or convex bodies (of a fixed area) in $\mathbb{S}_{+}^{2}$. The case of $\mathbb{H}^{2}$ might suggest that natural candidates for the maximizers are centered balls. However, this is not true. We will start with the following surprising result saying that centered balls in $\mathbb{S}_{+}^{2}$ are in fact the minimizers.

Theorem 5.1. Let $K$ be an origin-symmetric star body in $\mathbb{S}_{+}^{2}$. Then

$$
\int_{S^{1}} \operatorname{vol}\left(K \cap \xi^{\perp}\right)^{2} d \xi \geq 8 \pi \arccos ^{2}(1-\operatorname{vol}(K) / 2 \pi),
$$

with equality if and only if $K$ is a ball centered at the origin.

Proof. Let $B$ be the centered ball in $\mathbb{S}_{+}^{2}$ of the same volume as $K$, and let $r \in(0, \pi / 2)$ be the radius of $B$. Then

$$
\operatorname{vol}(K)=\int_{S^{1}} \int_{0}^{\rho_{K}(u)} \sin t d t d u=\int_{S^{1}}\left[1-\cos \left(\rho_{K}(u)\right)\right] d u
$$


and

$$
\operatorname{vol}(B)=\int_{S^{1}}\left[1-\cos \left(\rho_{B}(u)\right)\right] d u=2 \pi(1-\cos r),
$$

which yields $r=\arccos (1-\operatorname{vol}(K) / 2 \pi)$ and also

$$
\int_{S^{1}}\left[\cos r-\cos \left(\rho_{K}(u)\right)\right] d u=0 .
$$

The desired inequality comes from the following inequality. For any $x, r \in$ $(0, \pi / 2]$ we have

$$
x^{2}-r^{2} \geq \frac{2 r}{\sin r}(\cos r-\cos x),
$$

with equality if and only if $x=r$. Indeed, the function

$$
f(x)=x^{2}-r^{2}-\frac{2 r}{\sin r}(\cos r-\cos x)
$$

has the derivative

$$
f^{\prime}(x)=2 x-\frac{2 r}{\sin r} \sin x .
$$

The latter function is strictly convex in $(0, \pi / 2]$ and satisfies $f^{\prime}(0)=f^{\prime}(r)=$ 0 . Thus $f^{\prime}(x)<0$ when $0<x<r$ and $f^{\prime}(x)>0$ when $r<x<\pi / 2$. Therefore, $f$ has a strict minimum at $x=r$, i.e., $f(x)>f(r)=0$ for each $x \in(0, \pi / 2]$ with $x \neq r$. Finally we have

$$
\begin{aligned}
\int_{S^{1}} \operatorname{vol}\left(K \cap \xi^{\perp}\right)^{2} d \xi & -\int_{S^{1}} \operatorname{vol}\left(B \cap \xi^{\perp}\right)^{2} d \xi=4 \int_{S^{1}}\left[\rho_{K}^{2}(u)-r^{2}\right] d u \\
& \geq \frac{8 r}{\sin r} \int_{S^{1}}\left[\cos r-\cos \left(\rho_{K}(u)\right)\right] d u=0
\end{aligned}
$$

with the desired case of equality. The required inequality is now obtained by using $\operatorname{vol}\left(B \cap \xi^{\perp}\right)=2 \arccos (1-\operatorname{vol}(K) / 2 \pi)$.

We will now turn to the maximizers.

Theorem 5.2. Let $K \subset \mathbb{S}_{+}^{2}$ be origin-symmetric and star-shaped. Then

$$
\int_{S^{1}} \operatorname{vol}\left(K \cap \xi^{\perp}\right)^{2} d \xi \leq \pi^{2} \operatorname{vol}(K),
$$

with equality if and only if $K$ is a spherical cone.

Proof. Let $C$ be an origin-symmetric cone in $\mathbb{S}_{+}^{2}$ that has the same volume as $K$. Since $\rho_{C}$ takes the value $\frac{\pi}{2}$ on its support $A \subset S^{1}$, we get $\operatorname{vol}(C)=$ $\int_{A}\left[1-\cos \left(\frac{\pi}{2}\right)\right] d u=|A|$, and hence

$$
\int_{S^{1}} \operatorname{vol}\left(C \cap \xi^{\perp}\right)^{2} d \xi=4 \int_{S^{1}} \rho_{C}^{2}(\xi) d \xi=4(\pi / 2)^{2}|A|=\pi^{2} \operatorname{vol}(K) .
$$


Thus,

$$
\begin{aligned}
& \int_{S^{1}} \operatorname{vol}\left(C \cap \xi^{\perp}\right)^{2} d \xi-\int_{S^{1}} \operatorname{vol}\left(K \cap \xi^{\perp}\right)^{2} d \xi \\
& =\pi^{2} \int_{S^{1}}\left[1-\cos \left(\rho_{K}(u)\right)\right] d u-4 \int_{S^{1}} \rho_{K}^{2}(u) d u \\
& =2 \pi^{2} \int_{S^{1}}\left(\left[\sin \left(\rho_{K}(u) / 2\right)\right]^{2}-\left[(\sqrt{2} / \pi) \rho_{K}(u)\right]^{2}\right) d u .
\end{aligned}
$$

Finally, the inequality

$$
\sin (x / 2) \geq(\sqrt{2} / \pi) x, \quad 0 \leq x \leq \pi / 2,
$$

with its equality case, $x=0$ or $x=\pi / 2$, completes the proof.

In the next theorem we obtain an analogue of the previous result in the class of origin-symmetric convex bodies in $\mathbb{S}_{+}^{2}$. Here the maximizers are different; they are origin-symmetric lunes.

Theorem 5.3. Let $K$ be an origin-symmetric convex body in $\mathbb{S}_{+}^{2}$. Then

$$
\int_{S^{1}} \operatorname{vol}\left(K \cap \xi^{\perp}\right)^{2} d \xi \leq 16 \int_{0}^{\pi / 2} \arctan ^{2}\left(\frac{\tan (\operatorname{vol}(K) / 4)}{\cos \theta}\right) d \theta
$$

with equality if and only if $K$ is a lune.

To prove this theorem we need several auxiliary results. First, let us introduce some definitions. If $u$ and $v$ are two vectors on $S^{1}$, then $[u, v]$ stands for the segment of $S^{1}$ connecting $u$ and $v$, traced in the counterclockwise direction starting from $u$. By cone $(u, v)$ we denote the spherical cone with base $[u, v] \subset S^{1}$. In particular, if $u=v$, then cone $(u, u)$ stands for the geodesic ray from the origin in the direction of $u \in S^{1}$.

Let $x_{0} \in S^{1}$, and $K \subset \mathbb{S}_{+}^{2}$ be such that the interior of $K$ is connected and has the same volume as $K$. For our purposes $K$ will be a connected component of the difference of two star bodies. Define the function $f$ by

$$
f(x)=\frac{\operatorname{vol}\left(K \cap \operatorname{cone}\left(x_{0}, x\right)\right)}{\operatorname{vol}(K)}, \quad x \in D,
$$

where $D \subset S^{1}$ is the closure of the set of all $x$ 's such that the ray cone $(x, x)$ intersects the interior of $K$. Choosing $x_{0} \in S^{1} \backslash D$ if $S^{1} \backslash D \neq \emptyset$ or choosing any $x_{0} \in S^{1}$ otherwise, one can see that the function $f$ becomes injective. In this case the inverse function $f^{-1}:[0,1] \rightarrow S^{1}$ is called the inverse angular area function of $K$ measured counterclockwise from $x_{0}$. Note that $f$ and $f^{-1}$ do not depend on the choice of $x_{0} \in S^{1} \backslash D$ if $S^{1} \backslash D \neq \emptyset$. So in such situations we will just use the term the inverse angular area without specifying a direction $x_{0}$, but assuming that it lies outside of $D$.

Lemma 5.4. Let $K, \tilde{K}$ be star bodies in $\mathbb{S}_{+}^{2}$ such that $\tilde{K} \backslash K$ and $K \backslash \tilde{K}$ are connected and have equal positive volume. Let $\zeta^{+}, \zeta^{-}$be the inverse angular 
area functions of $\tilde{K} \backslash K, K \backslash \tilde{K}$ correspondingly. Then

$$
\int_{S^{1}}\left[\rho_{\tilde{K}}^{2}-\rho_{K}^{2}\right](\theta) d \theta>\operatorname{vol}(K \backslash \tilde{K}) \int_{0}^{1}\left[F\left(\rho_{K}\left(\zeta^{+}(t)\right)\right)-F\left(\rho_{K}\left(\zeta^{-}(t)\right)\right)\right] d t,
$$

where $F(x)=2 x / \sin x$. In particular, if

$$
\rho_{K}\left(\zeta^{+}(t)\right) \geq \rho_{K}\left(\zeta^{-}(t)\right), \quad t \in[0,1]
$$

then

$$
\int_{S^{1}} \rho_{\tilde{K}}^{2}(\theta) d \theta>\int_{S^{1}} \rho_{K}^{2}(\theta) d \theta
$$

Proof. Let both inverse angular area functions $\zeta^{+}, \zeta^{-}$be measured counterclockwise from some point $x_{0}$ on $S^{1}$ identified with zero in $[0,2 \pi)$. Note that

$$
\operatorname{vol}(\tilde{K} \backslash K)=\int_{0}^{2 \pi}\left[\left(1-\cos \rho_{\tilde{K}}\right)-\left(1-\cos \rho_{K}\right)\right]_{+} d \theta=\int_{0}^{2 \pi} g_{+}(\theta) d \theta
$$

and similarly

$$
\operatorname{vol}(K \backslash \tilde{K})=\int_{0}^{2 \pi} g_{-}(\theta) d \theta,
$$

where $g=\cos \rho_{K}-\cos \rho_{\tilde{K}}$ and $g_{+}=\max (g, 0), g_{-}=\max (-g, 0)$. Furthermore, for each $t \in[0,1]$,

$$
\int_{0}^{\zeta^{+}(t)} g_{+}(\theta) d \theta=t \operatorname{vol}(\tilde{K} \backslash K)
$$

and

$$
\int_{0}^{\zeta^{-(}(t)} g_{-}(\theta) d \theta=t \operatorname{vol}(K \backslash \tilde{K})
$$

The functions $\zeta^{+}, \zeta^{-}$are increasing and $\operatorname{vol}(K \backslash \tilde{K})=\operatorname{vol}(\tilde{K} \backslash K)$, so the above equalities can be written in a differential form:

$$
g_{+}\left(\zeta^{+}\right) d \zeta^{+}=\operatorname{vol}(K \backslash \tilde{K}) d t \quad \text { and } \quad g_{-}\left(\zeta^{+}\right) d \zeta^{-}=\operatorname{vol}(K \backslash \tilde{K}) d t .
$$

Using inequality (8), we have

$$
\begin{array}{r}
\int_{0}^{2 \pi}\left[\rho_{\tilde{K}}^{2}(\theta)-\rho_{K}^{2}(\theta)\right] d \theta>\int_{0}^{2 \pi} \frac{2 \rho_{K}(\theta)}{\sin \rho_{K}(\theta)}\left[\cos \rho_{K}(\theta)-\cos \rho_{\tilde{K}}(\theta)\right] d \theta \\
=\int_{\operatorname{supp}\left(g_{+}\right)} F\left(\rho_{K}(\theta)\right) g_{+}(\theta) d \theta-\int_{\operatorname{supp}\left(g_{-}\right)} F\left(\rho_{K}(\theta)\right) g_{-}(\theta) d \theta
\end{array}
$$

where $F(x)=2 x / \sin x$. The above inequality is strict because $K$ and $\tilde{K}$ are different. Also, the function $F$ is strictly increasing on $(0, \pi / 2)$ because $F^{\prime}(x)=2 \cos x(\tan x-x) / \sin ^{2} x$ is positive for each $x \in(0, \pi / 2)$. Changing 
variables $\theta=\zeta^{ \pm}(t)$ for each integral in the right-hand side of the equality above and using (9), we get

$$
\begin{aligned}
\int_{\operatorname{supp}\left(g_{+}\right)} F\left(\rho_{K}(\theta)\right) g_{+}(\theta) d \theta & =\int_{0}^{1} F\left(\rho_{K}\left(\zeta^{+}(t)\right)\right) g_{+}\left(\zeta^{+}(t)\right) d \zeta^{+}(t) \\
& =\operatorname{vol}(K \backslash \tilde{K}) \int_{0}^{1} F\left(\rho_{K}\left(\zeta^{+}(t)\right)\right) d t
\end{aligned}
$$

and

$$
\begin{aligned}
\int_{\operatorname{supp}\left(g_{-}\right)} F\left(\rho_{K}(\theta)\right) g_{-}(\theta) d \theta & =\int_{0}^{1} F\left(\rho_{K}\left(\zeta^{-}(t)\right)\right) g_{-}\left(\zeta^{-}(t)\right) d \zeta^{-}(t) \\
& =\operatorname{vol}(K \backslash \tilde{K}) \int_{0}^{1} F\left(\rho_{K}\left(\zeta^{-}(t)\right)\right) d t
\end{aligned}
$$

which completes the proof.

Below when we say that we take a point on a line (or, more generally, on a curve) $\ell$ in the direction of a vector $u \in S^{1}$, it means that we consider the geodesic ray emanating from the origin in the direction of $u$ and take the point of intersection of this geodesic ray and the line $\ell$.

Lemma 5.5. Let $K, L \subset \mathbb{S}_{+}^{2}$ be convex bodies that lie outside of a centered ball of radius $r>0$ and such that $\operatorname{vol}(K \cap L) \neq 0$. Then the inverse angular area functions $\zeta_{K}, \zeta_{L}$ of $K, L$ satisfy

$$
\left\|\zeta_{K}-\zeta_{L}\right\|_{C[0,1]}^{2} \leq c \frac{\operatorname{vol}(K \Delta L)}{\operatorname{vol}(K \cap L)}
$$

where $c=c(r)>0$ and $K \Delta L=(K \backslash L) \cup(L \backslash K)$.

Proof. If

$$
\frac{\operatorname{vol}(K \Delta L)}{\operatorname{vol}(K \cap L)}>\frac{1}{2}
$$

then we are done since $\left\|\zeta_{K}-\zeta_{L}\right\|_{C[0,1]}^{2} \leq\left(\left\|\zeta_{K}\right\|+\left\|\zeta_{L}\right\|\right)^{2}=4$ and we can take $c=8$. Thus, it is enough to consider the case $\operatorname{vol}(K \Delta L) / \operatorname{vol}(K \cap L) \leq$ $1 / 2$. We will assume that $\zeta_{K}, \zeta_{L}$ are measured counterclockwise from some point $x_{0} \in S^{1}$, such that the geodesic ray in this direction does not intersect $K \cup L$. As before, we will identify $S^{1}$ with $[0,2 \pi)$, and $x_{0}$ with $0 \in[0,2 \pi)$.

First, suppose that $\zeta_{K}(t) \geq \zeta_{L}(t)$ for a fixed $t \in[0,1]$. Then

$$
\operatorname{vol}\left[K \cap \operatorname{cone}\left(0, \zeta_{K}(t)\right)\right]=t \operatorname{vol}(K)
$$

and

$$
\begin{aligned}
\operatorname{vol}\left[K \cap \operatorname{cone}\left(0, \zeta_{L}(t)\right)\right] & \geq \operatorname{vol}\left[L \cap \operatorname{cone}\left(0, \zeta_{L}(t)\right)\right]-\operatorname{vol}(L \backslash K) \\
& =t \operatorname{vol}(L)-\operatorname{vol}(L \backslash K),
\end{aligned}
$$


so

$$
\begin{aligned}
& \operatorname{vol}\left[K \cap \operatorname{cone}\left(\zeta_{L}(t), \zeta_{K}(t)\right)\right] \\
& =\operatorname{vol}\left[K \cap \operatorname{cone}\left(0, \zeta_{K}(t)\right)\right]-\operatorname{vol}\left[K \cap \operatorname{cone}\left(0, \zeta_{L}(t)\right)\right] \\
& \leq t \operatorname{vol}(K)-t \operatorname{vol}(L)+\operatorname{vol}(L \backslash K) \\
& \leq \operatorname{vol}(K \Delta L) .
\end{aligned}
$$

Using (10) and the assumption $\operatorname{vol}(K \cap L) \geq 2 \operatorname{vol}(K \Delta L)$, we get

$$
\begin{aligned}
\operatorname{vol}\left[K \backslash \operatorname{cone}\left(\zeta_{L}(t), \zeta_{K}(t)\right)\right] & =\operatorname{vol}(K)-\operatorname{vol}\left[K \cap \operatorname{cone}\left(\zeta_{L}(t), \zeta_{K}(t)\right)\right] \\
& \geq \operatorname{vol}(K \cap L)-\operatorname{vol}(K \Delta L) \geq \operatorname{vol}(K \Delta L) \\
& \geq \operatorname{vol}\left[K \cap \operatorname{cone}\left(\zeta_{L}(t), \zeta_{K}(t)\right)\right] .
\end{aligned}
$$

Let $p_{0}, p_{1} \in \partial K$ be in the direction of $\zeta_{K}(t)$ with $\mathrm{d}\left(p_{0}, o\right) \leq \mathrm{d}\left(p_{1}, o\right)$ and $q_{0}, q_{1} \in \partial K$ be in the direction of $\zeta_{L}(t)$ with $\mathrm{d}\left(q_{0}, o\right) \leq \mathrm{d}\left(q_{1}, o\right)$. Consider the region $S$ enclosed by two lines through $p_{0}, q_{0}$ and through $p_{1}, q_{1}$. Then convexity of $K$ implies

$$
\begin{aligned}
\operatorname{vol}\left[K \cap \operatorname{cone}\left(\zeta_{L}(t), \zeta_{K}(t)\right)\right] & \geq \operatorname{vol}\left[S \cap \operatorname{cone}\left(\zeta_{L}(t), \zeta_{K}(t)\right)\right], \\
\operatorname{vol}\left[K \backslash \operatorname{cone}\left(\zeta_{L}(t), \zeta_{K}(t)\right)\right] & \leq \operatorname{vol}\left[S \backslash \operatorname{cone}\left(\zeta_{L}(t), \zeta_{K}(t)\right)\right],
\end{aligned}
$$

and hence

$$
\begin{aligned}
\operatorname{vol}(K) & =\operatorname{vol}\left[K \cap \operatorname{cone}\left(\zeta_{L}(t), \zeta_{K}(t)\right)\right]+\operatorname{vol}\left[K \backslash \operatorname{cone}\left(\zeta_{L}(t), \zeta_{K}(t)\right)\right] \\
& \leq 2 \operatorname{vol}\left[K \backslash \operatorname{cone}\left(\zeta_{L}(t), \zeta_{K}(t)\right)\right] \leq 2 \operatorname{vol}\left[S \backslash \operatorname{cone}\left(\zeta_{L}(t), \zeta_{K}(t)\right)\right] \\
& \leq 2 \operatorname{vol}(S) .
\end{aligned}
$$

Thus,

$$
\frac{\operatorname{vol}\left[K \cap \operatorname{cone}\left(\zeta_{L}(t), \zeta_{K}(t)\right)\right]}{\operatorname{vol}(K)} \geq \frac{\operatorname{vol}\left[S \cap \operatorname{cone}\left(\zeta_{L}(t), \zeta_{K}(t)\right)\right]}{2 \operatorname{vol}(S)} .
$$

We will now bound the right-hand side of 12$)$. By $\ell(\theta)$ we will denote the line passing through the point $o_{*}=p_{0} q_{0} \cap p_{1} q_{1}$ and making an angle $\theta$ with the line $p_{0} q_{0}$.

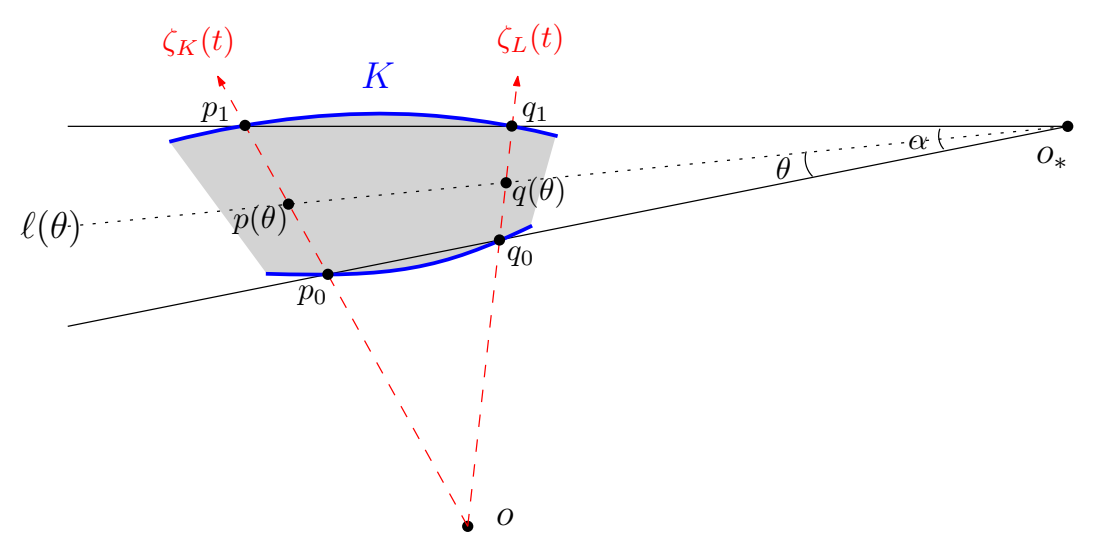


Let $p(\theta), q(\theta) \in \ell(\theta)$ be the points in the direction of $\zeta_{K}(t), \zeta_{L}(t)$ respectively. If $\alpha$ is the angle between the lines through $p_{0}, q_{0}$ and through $p_{1}, q_{1}$, then $\operatorname{vol}(S)=2 \alpha$ and

$$
\begin{aligned}
\operatorname{vol}\left[S \cap \operatorname{cone}\left(\zeta_{L}(t), \zeta_{K}(t)\right)\right] & =\int_{0}^{\alpha}\left|\cos \mathrm{d}\left(p(\theta), o_{*}\right)-\cos \mathrm{d}\left(q(\theta), o_{*}\right)\right| d \theta \\
& \geq \frac{2}{\pi^{2}} \int_{0}^{\alpha} \mathrm{d}(p(\theta), q(\theta))^{2} d \theta .
\end{aligned}
$$

The last inequality comes from

$$
\frac{|\cos x-\cos y|}{2}=\sin \left(\frac{x+y}{2}\right)\left|\sin \left(\frac{x-y}{2}\right)\right| \geq \sin ^{2}\left(\frac{x-y}{2}\right) \geq\left(\frac{x-y}{\pi}\right)^{2},
$$

for $x, y \in[0, \pi / 2]$. Since, by the hypothesis of the lemma, $p(\theta), q(\theta) \in K$ are at least distance $r$ from the origin, we have

$$
\mathrm{d}(p(\theta), q(\theta))=\left|\ell(\theta) \cap \operatorname{cone}\left(\zeta_{L}(t), \zeta_{K}(t)\right)\right| \geq\left|\zeta_{K}(t)-\zeta_{L}(t)\right| \sin r .
$$

Thus

$$
\begin{aligned}
\frac{\operatorname{vol}\left[S \cap \operatorname{cone}\left(\zeta_{L}(t), \zeta_{K}(t)\right)\right]}{2 \operatorname{vol}(S)} & \geq \frac{\alpha\left(2 / \pi^{2}\right)\left|\zeta_{K}(t)-\zeta_{L}(t)\right|^{2} \sin ^{2} r}{4 \alpha} \\
& =\frac{\sin ^{2} r}{2 \pi^{2}}\left|\zeta_{K}(t)-\zeta_{L}(t)\right|^{2}
\end{aligned}
$$

Therefore, 10) and (12) imply

$$
\left|\zeta_{K}(t)-\zeta_{L}(t)\right|^{2} \leq \frac{2 \pi^{2}}{\sin ^{2} r} \cdot \frac{\operatorname{vol}(K \Delta L)}{\operatorname{vol}(K)},
$$

and similarly, in case of $\zeta_{K}(t) \leq \zeta_{L}(t)$,

$$
\left|\zeta_{K}(t)-\zeta_{L}(t)\right|^{2} \leq \frac{2 \pi^{2}}{\sin ^{2} r} \cdot \frac{\operatorname{vol}(K \Delta L)}{\operatorname{vol}(L)},
$$

which completes the proof.

We will adopt the following notation. Each geodesic line $\ell$ in $\mathbb{S}_{+}^{2}$ not passing through the origin cuts $\mathbb{S}_{+}^{2}$ into two open sets with the common boundary $\ell$. These sets will be denoted by $H_{\ell}^{+}$and $H_{\ell}^{-}$, where $H_{\ell}^{-}$contains the origin and $H_{\ell}^{+}$does not.

Lemma 5.6. Let $K \subset \mathbb{S}_{+}^{2}$ be a convex body and $\ell \subset \mathbb{S}_{+}^{2}$ be a line intersecting the interior of $K$, but not containing the origin. Consider a family of lines $\ell_{\varepsilon}$ in $\mathbb{S}_{+}^{2}$ meeting $\ell$ at an angle $\varepsilon$ (where $\varepsilon>0$ is small enough) such that

$$
K_{\varepsilon}=K \cap H_{\ell}^{-} \cap H_{\ell_{\varepsilon}}^{+} \quad\left(\text { or } K_{\varepsilon}=K \cap H_{\ell}^{+} \cap H_{\ell_{\varepsilon}}^{-}\right)
$$

is of positive measure and $\ell \cap \ell_{\varepsilon}$ converges to a point $o_{*} \in \ell$ as $\varepsilon \rightarrow 0$. Then the inverse angular area function $\zeta_{\varepsilon}$ of $K_{\varepsilon}$ converges uniformly, as $\varepsilon \rightarrow 0$, to a function $\zeta:[0,1] \rightarrow S^{1}$ such that for each $t \in[0,1]$

$$
\cos \mathrm{d}\left(P_{\ell} \zeta(t), o_{*}\right)=(1-t) \cos \mathrm{d}\left(P_{\ell} \zeta(0), o_{*}\right)+t \cos \mathrm{d}\left(P_{\ell} \zeta(1), o_{*}\right) .
$$


Here, $P_{\ell} \xi$ denotes the point on $\ell$ in the direction of $\xi \in S^{1}$.

Proof. Let $K_{\varepsilon}=K \cap H_{\ell}^{-} \cap H_{\ell_{\varepsilon}}^{+}$be of positive measure for small $\varepsilon>0$. The same argument works for the case of $K_{\varepsilon}=K \cap H_{\ell}^{+} \cap H_{\ell_{\varepsilon}}^{-}$.

For $0 \leq \theta \leq \varepsilon$, we denote by $\ell_{\varepsilon}(\theta)$ the line through $o_{\varepsilon}=\ell \cap \ell_{\varepsilon}$ that intersects $\ell$ at an angle $\theta$ and also intersects $K_{\varepsilon}$. (On the picture below the point $o_{\varepsilon}$ is located outside of $K$, but in the proof there is no restriction on the position of $\left.o_{\varepsilon}\right)$. For each $t \in[0,1]$ and $\theta \in[0, \varepsilon]$, consider the point $p_{t, \varepsilon}(\theta)$ on the line $\ell_{\varepsilon}(\theta)$ in the direction of $\zeta_{\varepsilon}(t)$. Let $q_{t, \varepsilon}(\theta)$ be the same point as $p_{t, \varepsilon}(\theta)$ if $p_{t, \varepsilon}(\theta) \in K$, and let $q_{t, \varepsilon}(\theta)$ be the endpoint of $K \cap \ell_{\varepsilon}(\theta)$ closest to $p_{t, \varepsilon}(\theta)$ if $p_{t, \varepsilon}(\theta) \notin K$. In case of $o_{\varepsilon} \notin K$, the following figure shows where these points are located. If $o_{\varepsilon} \in K$, all $p_{0, \varepsilon}(\theta)$ and $q_{0, \varepsilon}(\theta)$ indicate the common point $o_{\varepsilon}$.

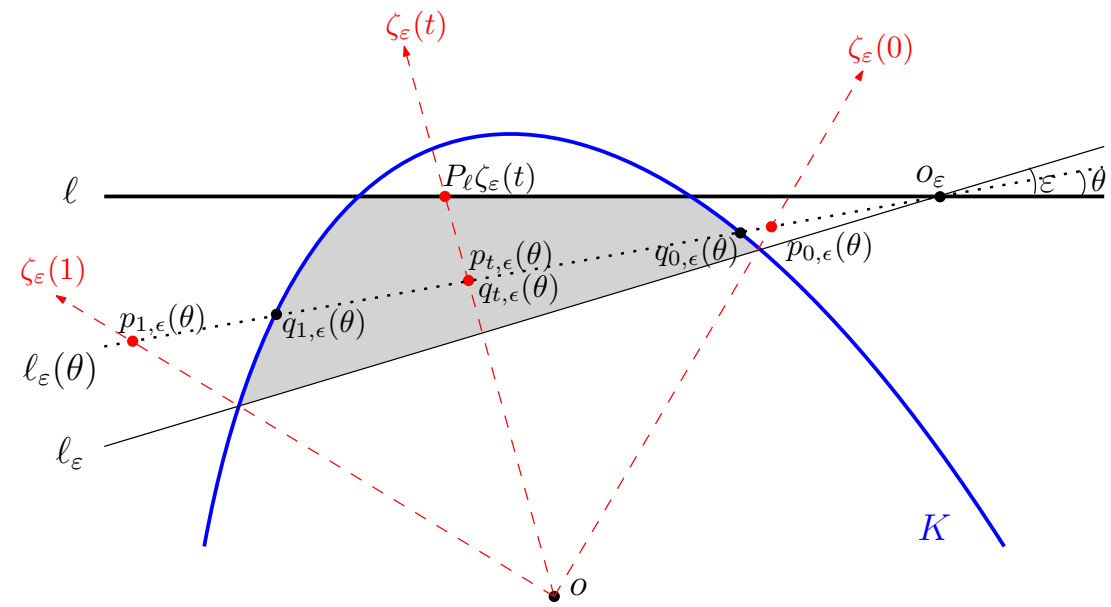

Then the areas that appear in the definition of $\zeta_{\varepsilon}$ can be computed by considering a polar coordinate system centered at $o_{\varepsilon}$. For all $t \in[0,1]$ we have

$$
\begin{aligned}
& \int_{0}^{\varepsilon}\left(\left[1-\cos \mathrm{d}\left(q_{t, \varepsilon}(\theta), o_{\varepsilon}\right)\right]-\left[1-\cos \mathrm{d}\left(q_{0, \varepsilon}(\theta), o_{\varepsilon}\right)\right]\right) d \theta \\
& =t \int_{0}^{\varepsilon}\left(\left[1-\cos \mathrm{d}\left(q_{1, \varepsilon}(\theta), o_{\varepsilon}\right)\right]-\left[1-\cos \mathrm{d}\left(q_{0, \varepsilon}(\theta), o_{\varepsilon}\right)\right]\right) d \theta .
\end{aligned}
$$

Rearranging, we have

$$
\begin{aligned}
& \frac{1}{\varepsilon} \int_{0}^{\varepsilon} \cos \mathrm{d}\left(q_{t, \varepsilon}(\theta), o_{\varepsilon}\right) d \theta \\
& =\frac{1-t}{\varepsilon} \int_{0}^{\varepsilon} \cos \mathrm{d}\left(q_{0, \varepsilon}(\theta), o_{\varepsilon}\right) d \theta+\frac{t}{\varepsilon} \int_{0}^{\varepsilon} \cos \mathrm{d}\left(q_{1, \varepsilon}(\theta), o_{\varepsilon}\right) d \theta .
\end{aligned}
$$

We first show that

$$
f_{\varepsilon}(t)=\frac{1}{\varepsilon} \int_{0}^{\varepsilon} \cos \mathrm{d}\left(q_{t, \varepsilon}(\theta), o_{\varepsilon}\right) d \theta, \quad t \in[0,1],
$$


converges uniformly (in $t$ ) as $\varepsilon \rightarrow 0$. Since $f_{\varepsilon}(t)=(1-t) f_{\varepsilon}(0)+t f_{\varepsilon}(1)$, it suffices to show that both $f_{\varepsilon}(0)$ and $f_{\varepsilon}(1)$ converge as $\varepsilon \rightarrow 0$. Indeed, if $q_{0}$, $q_{1}$ are the endpoints of $K_{\varepsilon} \cap \ell$ with $\mathrm{d}\left(q_{0}, o_{*}\right)<\mathrm{d}\left(q_{1}, o_{*}\right)$, then

$$
\begin{aligned}
\left|f_{\varepsilon}(0)-\cos \mathrm{d}\left(q_{0}, o_{*}\right)\right| & \leq \frac{1}{\varepsilon} \int_{0}^{\varepsilon}\left|\cos \mathrm{d}\left(q_{0, \varepsilon}(\theta), o_{\varepsilon}\right)-\cos \mathrm{d}\left(q_{0}, o_{*}\right)\right| d \theta \\
& \leq \frac{1}{\varepsilon} \int_{0}^{\varepsilon}\left|\mathrm{d}\left(q_{0, \varepsilon}(\theta), o_{\varepsilon}\right)-\mathrm{d}\left(q_{0}, o_{*}\right)\right| \mathrm{d} \theta \\
& \leq \frac{1}{\varepsilon} \int_{0}^{\varepsilon}\left(\left|\mathrm{d}\left(q_{0, \varepsilon}(\theta), o_{\varepsilon}\right)-\mathrm{d}\left(q_{0}, o_{\varepsilon}\right)\right|+\mathrm{d}\left(o_{\varepsilon}, o_{*}\right)\right) d \theta \\
& \leq \mathrm{d}\left(q_{0, \varepsilon}(\varepsilon), q_{0}\right)+\mathrm{d}\left(o_{\varepsilon}, o_{*}\right),
\end{aligned}
$$

and similarly $\left|f_{\varepsilon}(1)-\cos \mathrm{d}\left(q_{1}, o_{*}\right)\right| \leq \mathrm{d}\left(q_{1, \varepsilon}(\varepsilon), q_{1}\right)+\mathrm{d}\left(o_{\varepsilon}, o_{*}\right)$. Since both $\mathrm{d}\left(q_{0, \varepsilon}(\varepsilon), q_{0}\right)$ and $\mathrm{d}\left(q_{1, \varepsilon}(\varepsilon), q_{1}\right)$ approach 0 as $\varepsilon \rightarrow 0$, it follows that $f_{\varepsilon}(0)$ converges to $\cos \mathrm{d}\left(q_{0}, o_{*}\right)$, and $f_{\varepsilon}(1)$ converges to $\cos \mathrm{d}\left(q_{1}, o_{*}\right)$.

Let $f$ be the limit of $f_{\varepsilon}$ as $\varepsilon \rightarrow 0$. Define the function $\zeta:[0,1] \rightarrow S^{1}$ by

$$
\cos \mathrm{d}\left(P_{\ell} \zeta(t), o_{*}\right)=f(t), \quad t \in[0,1] .
$$

Observing that $P_{\ell} \zeta_{\varepsilon}(t)=p_{t, \varepsilon}(0)$, we have

$$
\begin{aligned}
& \left|\cos \mathrm{d}\left(P_{\ell} \zeta_{\varepsilon}(t), o_{*}\right)-\cos \mathrm{d}\left(P_{\ell} \zeta(t), o_{*}\right)\right| \\
& \leq\left|\cos \mathrm{d}\left(P_{\ell} \zeta_{\varepsilon}(t), o_{*}\right)-f_{\varepsilon}(t)\right|+\left\|f_{\varepsilon}-f\right\|_{\infty} \\
& =\frac{1}{\varepsilon}\left|\int_{0}^{\varepsilon}\left[\cos \mathrm{d}\left(p_{t, \varepsilon}(0), o_{*}\right)-\cos \mathrm{d}\left(q_{t, \varepsilon}(\theta), o_{\varepsilon}\right)\right] d \theta\right|+\left\|f_{\varepsilon}-f\right\|_{\infty} \\
& \leq \frac{1}{\varepsilon} \int_{0}^{\varepsilon} \mathrm{d}\left(p_{t, \varepsilon}(0), q_{t, \varepsilon}(\theta)\right) d \theta+\mathrm{d}\left(o_{\varepsilon}, o_{*}\right)+\left\|f_{\varepsilon}-f\right\|_{\infty} .
\end{aligned}
$$

To show the uniform convergence of $\zeta_{\varepsilon}$ to $\zeta$, it suffices to find a positive constant $c=c(K, \ell)$ such that

$$
\mathrm{d}\left(p_{t, \varepsilon}(0), q_{t, \varepsilon}(\theta)\right) \leq c \varepsilon, \quad 0 \leq t \leq 1, \quad 0 \leq \theta \leq \varepsilon .
$$

Indeed, if $p_{t, \varepsilon}(\theta) \in K$, then $q_{t, \varepsilon}(\theta)=p_{t, \varepsilon}(\theta)$ and hence

$$
\mathrm{d}\left(p_{t, \varepsilon}(0), q_{t, \varepsilon}(\theta)\right)=\mathrm{d}\left(p_{t, \varepsilon}(0), p_{t, \varepsilon}(\theta)\right) \leq \mathrm{d}\left(p_{t, \varepsilon}(0), p_{t, \varepsilon}(\varepsilon)\right) .
$$

Otherwise, $q_{t, \varepsilon}(\theta)$ is on the boundary of $K$, so we get

$$
\begin{aligned}
\mathrm{d}\left(p_{t, \varepsilon}(0), q_{t, \varepsilon}(\theta)\right) & \leq \mathrm{d}\left(p_{t, \varepsilon}(0), \bar{p}\right)+\mathrm{d}\left(\bar{p}, q_{t, \varepsilon}(\theta)\right) \\
& \leq \mathrm{d}\left(p_{t, \varepsilon}(0), p_{t, \varepsilon}(\varepsilon)\right)+\mathrm{d}\left(\ell \cap \ell_{0}, \ell_{\varepsilon} \cap \ell_{0}\right),
\end{aligned}
$$

where $\ell_{0}$ is a supporting line to $K$ at $q_{t, \varepsilon}(\theta)$ and $\bar{p}$ is the point on the line $\ell_{0}$ in the direction of $\zeta_{\varepsilon}(t)$.

Then the law of sines for the triangle enclosed by $\ell, \ell_{\varepsilon}$ and the direction of $\zeta_{\varepsilon}(t)$ gives

$$
\sin \mathrm{d}\left(p_{t, \varepsilon}(0), p_{t, \varepsilon}(\varepsilon)\right)=\sin \mathrm{d}\left(p_{t, \varepsilon}(\varepsilon), o_{\varepsilon}\right) \cdot \frac{\sin \varepsilon}{\sin \alpha} \leq \frac{\sin \varepsilon}{\sin \alpha},
$$




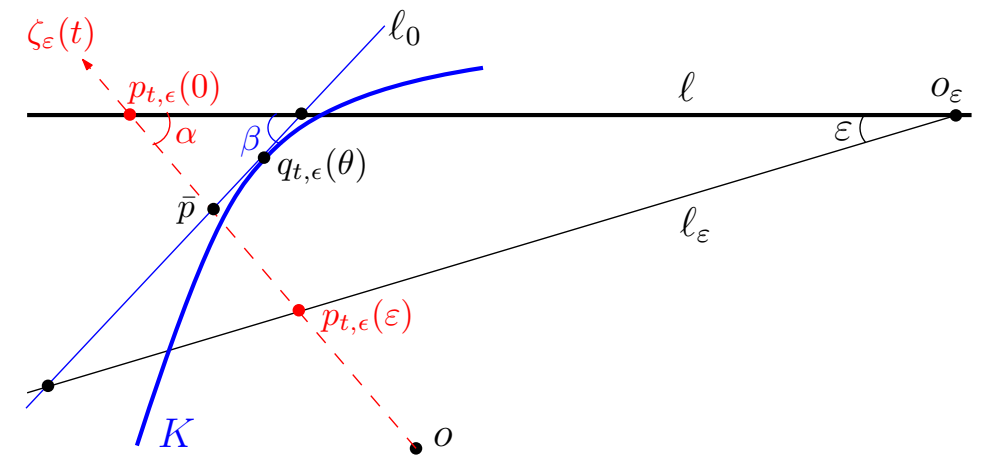

and for the triangle enclosed by $\ell, \ell_{\varepsilon}$ and $\ell_{0}$,

$$
\sin \mathrm{d}\left(\ell \cap \ell_{0}, \ell_{\varepsilon} \cap \ell_{0}\right)=\sin \mathrm{d}\left(\ell_{\varepsilon} \cap \ell_{0}, o_{\varepsilon}\right) \cdot \frac{\sin \varepsilon}{\sin \beta} \leq \frac{\sin \varepsilon}{\sin \beta} .
$$

Here $\alpha \in[0, \pi / 2]$ is the angle between the line $\ell$ and the direction $\zeta_{\varepsilon}(t)$, which is bounded below by a positive constant depending on $K \cap \ell$ only. Similarly, the angle $\beta$ between $\ell_{0}$ and $\ell$ is bounded below by a constant depending only on $K$ and $\ell$. Thus both $\mathrm{d}\left(p_{t, \varepsilon}(0), p_{t, \varepsilon}(\varepsilon)\right)$ and $\mathrm{d}\left(\ell \cap \ell_{0}, \ell_{\varepsilon} \cap \ell_{0}\right)$ are less than a constant multiple of $\varepsilon$, and so is $\mathrm{d}\left(p_{t, \varepsilon}(0), q_{t, \varepsilon}(\theta)\right)$ in either case.

Therefore, $\zeta_{\varepsilon}$ uniformly converges to $\zeta$, and we can get the desired equality for $\zeta$ by letting $\varepsilon \rightarrow 0$ in 13 .

Lemma 5.7. Let $K$ be a convex body in $\mathbb{S}_{+}^{2}$ and $\ell$ a line in $\mathbb{S}_{+}^{2}$ intersecting the interior of $K$. For each small $\varepsilon>0$, choose a line $\ell_{\varepsilon}$ that makes an angle $\varepsilon$ with $\ell$ and such that the regions of $K$ cut off by $\ell$ and $\ell_{\varepsilon}$ have equal areas. Then the point $\ell \cap \ell_{\varepsilon}$ converges to the midpoint of $K \cap \ell$ as $\varepsilon \rightarrow 0$.

Proof. We may assume that $\ell$ does not pass through the origin and $K \cap H_{\ell}^{+}$ has the same volume as $K \cap H_{\ell_{\varepsilon}}^{+}$. For $\theta \in[0, \varepsilon]$, we denote by $\ell_{\varepsilon}(\theta)$ the line that passes through $o_{\varepsilon}=\ell \cap \ell_{\varepsilon}$, makes an angle $\theta$ with $\ell$, and intersects $H_{\ell}^{+} \cap H_{\ell_{\varepsilon}}^{-}$.

Let $p_{\varepsilon}(\theta), q_{\varepsilon}(\theta)$ be the endpoints of $K \cap \ell_{\varepsilon}(\theta)$ such that $p_{\varepsilon}(\theta) \in H_{\ell}^{+}$and $q_{\varepsilon}(\theta) \in H_{\ell}^{-}$. In particular, if $\theta=0$, then $p_{\varepsilon}(0)$ and $q_{\varepsilon}(0)$ are the endpoints of the interval $K \cap \ell$. Write them as $p_{0}=p_{\varepsilon}(0)$ and $q_{0}=q_{\varepsilon}(0)$. Then

$$
\begin{aligned}
0 & =\frac{1}{\varepsilon}\left(\operatorname{vol}\left(K \cap H_{\ell}^{+}\right)-\operatorname{vol}\left(K \cap H_{\ell_{\varepsilon}}^{+}\right)\right) \\
& =\frac{1}{\varepsilon} \int_{0}^{\varepsilon}\left(\left[1-\cos \mathrm{d}\left(p_{\varepsilon}(\theta), o_{\varepsilon}\right)\right]-\left[1-\cos \mathrm{d}\left(q_{\varepsilon}(\theta), o_{\varepsilon}\right)\right]\right) d \theta \\
& =\frac{1}{\varepsilon} \int_{0}^{\varepsilon}\left[\cos \mathrm{d}\left(q_{\varepsilon}(\theta), o_{\varepsilon}\right)-\cos \mathrm{d}\left(p_{\varepsilon}(\theta), o_{\varepsilon}\right)\right] d \theta \\
& =\cos \mathrm{d}\left(q_{0}, o_{\varepsilon}\right)-\cos \mathrm{d}\left(p_{0}, o_{\varepsilon}\right)+g(\varepsilon)-f(\varepsilon)
\end{aligned}
$$


where

$$
\begin{aligned}
|f(\varepsilon)| & =\frac{1}{\varepsilon}\left|\int_{0}^{\varepsilon}\left[\cos \mathrm{d}\left(p_{\varepsilon}(\theta), o_{\varepsilon}\right)-\cos \mathrm{d}\left(p_{0}, o_{\varepsilon}\right)\right] d \theta\right| \\
& \leq \frac{1}{\varepsilon} \int_{0}^{\varepsilon}\left|\mathrm{d}\left(p_{\varepsilon}(\theta), o_{\varepsilon}\right)-\mathrm{d}\left(p_{0}, o_{\varepsilon}\right)\right| d \theta \leq \mathrm{d}\left(p_{\varepsilon}(\varepsilon), p_{0}\right)
\end{aligned}
$$

and

$$
|g(\varepsilon)|=\frac{1}{\varepsilon}\left|\int_{0}^{\varepsilon}\left[\cos \mathrm{d}\left(q_{\varepsilon}(\theta), o_{\varepsilon}\right)-\cos \mathrm{d}\left(q_{0}, o_{\varepsilon}\right)\right] d \theta\right| \leq \mathrm{d}\left(q_{\varepsilon}(\varepsilon), q_{0}\right) .
$$

Since $\mathrm{d}\left(p_{\varepsilon}(\varepsilon), p_{0}\right) \rightarrow 0$ and $\mathrm{d}\left(q_{\varepsilon}(\varepsilon), q_{0}\right) \rightarrow 0$ as $\varepsilon \rightarrow 0$, we have that

$$
\cos \mathrm{d}\left(p_{0}, o_{\varepsilon}\right)-\cos \mathrm{d}\left(q_{0}, o_{\varepsilon}\right)=g(\varepsilon)-f(\varepsilon)
$$

converges to 0 as $\varepsilon \rightarrow 0$. This implies that $o_{\varepsilon}$ converges to the midpoint of $p_{0}$ and $q_{0}$ as $\varepsilon \rightarrow 0$.

Proof of Theorem 5.3. First we claim that there is a body that maximizes the integral $\int_{S^{1}} \rho_{K}^{2}(\theta) d \theta$ in the class of origin-symmetric convex bodies in $\mathbb{S}_{+}^{2}$ of a fixed volume. To see this, we can identify convex bodies in $\mathbb{S}_{+}^{2}$ with convex bodies in $\mathbb{R}^{3}$ that are obtained as the intersections of the unit ball $B^{3}$ and convex cones in $\mathbb{R}^{3}$ with vertex at the origin. The claim now follows from the Blaschke selection principle.

Suppose that there exists a body $K$ that is not a lune and that maximizes $\int_{S^{1}} \rho_{K}^{2}(\theta) d \theta$ in the class of origin-symmetric convex bodies in $\mathbb{S}_{+}^{2}$ of a fixed volume. (We disregard the trivial case when the volume is equal to $\operatorname{vol}\left(\mathbb{S}_{+}^{2}\right)$ ). To get a contradiction we will construct an origin-symmetric convex body $\tilde{K} \subset \mathbb{S}_{+}^{2}$ satisfying $\operatorname{vol}(K)=\operatorname{vol}(\tilde{K})$ and $\int_{S^{1}} \rho_{\tilde{K}}^{2}(\theta) d \theta>\int_{S^{1}} \rho_{K}^{2}(\theta) d \theta$. We will consider the following cases according to the type of the boundary of $K$. By $[u, v]$ we denote the geodesic line segment between two points $u, v \in \mathbb{S}_{+}^{2}$. A line segment $[u, v] \subset \mathbb{S}_{+}^{2}$ is called an edge of $K$ if it coincides with the intersection of the boundary of $K$ and the line through $u$ and $v$.

(1) $K$ has an arc on its boundary not containing any edges, i.e., the boundary of $K$ is strictly convex along the arc.

(2) $K$ has an edge $\left[p_{1}^{+}, p_{1}^{-}\right]$with $\mathrm{d}\left(p_{1}^{+}, o\right)>\mathrm{d}\left(p_{1}^{-}, o\right)$.

(3) $K$ has two edges $\left[p_{0}, p_{1}\right]$ and $\left[q_{0}, q_{1}\right]$ such that $\mathrm{d}\left(p_{0}, p_{1}\right)<\mathrm{d}\left(q_{0}, q_{1}\right)$ and $\mathrm{d}\left(p_{0}, o\right)=\mathrm{d}\left(p_{1}, o\right)=\mathrm{d}\left(q_{0}, o\right)=\mathrm{d}\left(q_{1}, o\right)=\max _{x \in K} \mathrm{~d}(x, o)$.

(4) None of the above. In this case $K$ is reduced to a regular spherical polygon.

It suffices to define such $\tilde{K}$ only in the half-space corresponding to $[0, \pi) \subset S^{1}$ and to assume that the arc and edges in the above cases lie on the half-space.

Case (1): In this case we can choose two disjoint intervals $U, V \subset[0, \pi)$ such that

$$
\max _{u \in U} \rho_{K}(u) \leq \min _{v \in V} \rho_{K}(v)
$$


and the boundary of $K$ is strictly convex in the directions corresponding to $U$ and $V$. Strict convexity enables us to construct a convex body $\tilde{K} \subset \mathbb{S}_{+}^{2}$

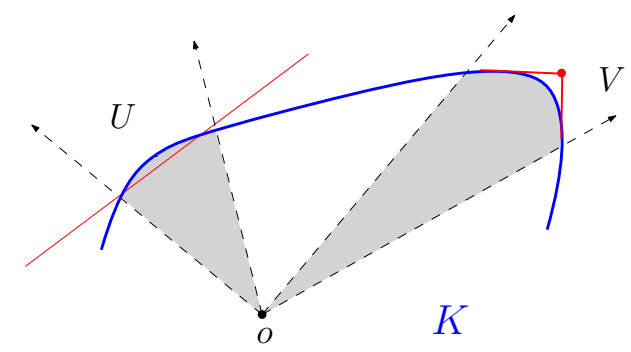

such that $\operatorname{vol}(K)=\operatorname{vol}(\tilde{K})$, and $\rho_{K}(x) \geq \rho_{\tilde{K}}(x)$ if $x \in U, \rho_{K}(x) \leq \rho_{\tilde{K}}(x)$ if $x \in V$, and $\rho_{K}(x)=\rho_{\tilde{K}}(x)$ otherwise. Such a body $\tilde{K}$ can be obtained from $K$ by cutting off by a straight line a small piece of $K$ that lies in the region corresponding to $U$, and then taking the convex hull of $K$ and a point outside of $K$ in the region corresponding to $V$. Moreover, we can ensure that the volumes of $K$ and $\tilde{K}$ are the same. Strict convexity guarantees that the boundaries of $K$ and $\tilde{K}$ can be different only in the regions corresponding to $U$ and $V$. Using the notation of Lemma 5.4, we see that $\zeta^{+}$and $\zeta^{-}$take on their values only in $V$ and $U$ respectively. Therefore, by (14) and Lemma 5.4 we have $\int_{S^{1}} \rho_{\tilde{K}}^{2} d \theta>\int_{S^{1}} \rho_{K}^{2}(\theta) d \theta$.

Case (2): Let $\ell$ be the line through $p_{1}^{+}$and $p_{1}^{-}$. We will consider two possibilities: when $\ell$ is the only supporting line to the body $K$ at $p_{1}^{+}$, and when it is not. If $\ell$ is the only supporting line to $K$ at $p_{1}^{+}$, we can use the same argument as in Case (1) to construct $\tilde{K}$. More precisely, we can cut off a small piece of $K$ in a neighborhood of $p_{1}^{-}$, and then take the convex hull with a point near $p_{1}^{+}$. The latter can be done as follows. Take a point $p$ on the line $\ell$ outside of $K$ and close to $p_{1}^{+}$. From this point $p$ draw the supporting line (different from $\ell$ ) to the body $K$. As $p$ approaches $p_{1}^{+}$, the supporting line approaches $\ell$ and the point of contact approaches $p_{1}^{+}$. Thus if we take such a point $p$ sufficiently close to $p_{1}^{+}$, and take the convex hull of $p$ and the body $K$, then the resulting body will differ from $K$ only in a small neighborhood of $p_{1}^{+}$. The rest of the proof goes as in Case (1).

Suppose now that there is another supporting line $\ell_{0}$ at $p_{1}^{+}$other than $\ell$. For small $\varepsilon>0$ choose a line $\ell_{\varepsilon}$ satisfying the following three conditions: (i) $\ell_{\varepsilon}$ separates $p_{1}^{-}$from $p_{1}^{+}$and the origin; (ii) the angle between $\ell$ and $\ell_{\varepsilon}$ is equal to $\varepsilon$; (iii) the area of $K_{\varepsilon}^{+}=H_{\ell_{0}}^{-} \cap H_{\ell}^{+} \cap H_{\ell_{\varepsilon}}^{-}$is equal to that of $K_{\varepsilon}^{-}=K \cap H_{\ell_{\varepsilon}}^{+}$.

Then the body $\tilde{K}$ is obtained from $K$ by adding $K_{\varepsilon}^{+}$and by removing $K_{\varepsilon}^{-}$. Let $\zeta_{\varepsilon}^{+}, \zeta_{\varepsilon}^{-}$be the inverse angular area functions of $K_{\varepsilon}^{+}, K_{\varepsilon}^{-}$measured from $\ell \cap \ell_{\varepsilon}$ in opposite directions. Then, as $\varepsilon \rightarrow 0$, the point $\ell \cap \ell_{\varepsilon}$ converges to the midpoint $p_{0}$ of $p_{1}^{+}$and $p_{1}^{-}$(Lemma 5.7), and for each choice of the sign \pm the function $\zeta_{\varepsilon}^{ \pm}$uniformly converges to $\zeta^{ \pm}$, by Lemma 5.6 , such that 


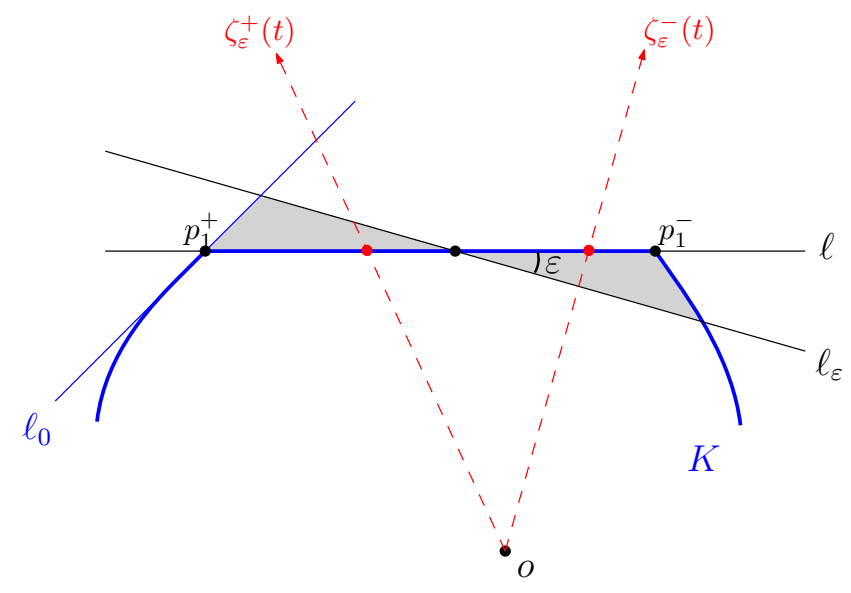

for each $t \in[0,1]$ the point $p_{t}^{ \pm} \in \ell$ in the direction of $\zeta^{ \pm}(t)$ satisfies

$$
\begin{aligned}
\cos \mathrm{d}\left(p_{t}^{ \pm}, p_{0}\right) & =(1-t) \cos \mathrm{d}\left(p_{0}^{ \pm}, p_{0}\right)+t \cos \mathrm{d}\left(p_{1}^{ \pm}, p_{0}\right) \\
& =1-t+t \cos \left(\mathrm{d}\left(p_{1}^{+}, p_{1}^{-}\right) / 2\right) .
\end{aligned}
$$

This implies that $\mathrm{d}\left(p_{t}^{+}, p_{0}\right)=\mathrm{d}\left(p_{t}^{-}, p_{0}\right)$ for all $t \in[0,1]$. For each $t \in[0,1]$ and each of the signs \pm , the law of cosines for the triangle with vertices $o$, $p_{0}$ and $p_{t}^{ \pm}$yields

$$
\cos \mathrm{d}\left(p_{t}^{ \pm}, o\right)=\cos \mathrm{d}\left(p_{0}, o\right) \cos \mathrm{d}\left(p_{t}^{ \pm}, p_{0}\right) \mp \sin \mathrm{d}\left(p_{0}, o\right) \sin \mathrm{d}\left(p_{t}^{ \pm}, p_{0}\right) \cos \gamma,
$$

where $\gamma$ is the angle between the lines through $o, p_{0}$ and through $p_{0}, p_{1}^{+}$. Note that $\gamma \neq \pi / 2$. Since $\mathrm{d}\left(p_{t}^{+}, p_{0}\right)=\mathrm{d}\left(p_{t}^{-}, p_{0}\right)$, the above equations imply

$$
\cos \mathrm{d}\left(p_{t}^{-}, o\right)-\cos \mathrm{d}\left(p_{t}^{+}, o\right)=2 \sin \mathrm{d}\left(p_{0}, o\right) \sin \mathrm{d}\left(p_{t}^{+}, p_{0}\right) \cos \gamma
$$

and therefore

$$
\begin{aligned}
\frac{\cos \mathrm{d}\left(p_{t}^{-}, o\right)-\cos \mathrm{d}\left(p_{t}^{+}, o\right)}{\cos \mathrm{d}\left(p_{1}^{-}, o\right)-\cos \mathrm{d}\left(p_{1}^{+}, o\right)} & =\frac{2 \sin \mathrm{d}\left(p_{0}, o\right) \sin \mathrm{d}\left(p_{t}^{+}, p_{0}\right) \cos \gamma}{2 \sin \mathrm{d}\left(p_{0}, o\right) \sin \mathrm{d}\left(p_{1}^{+}, p_{0}\right) \cos \gamma} \\
& =\frac{\sin \mathrm{d}\left(p_{t}^{+}, p_{0}\right)}{\sin \mathrm{d}\left(p_{1}^{+}, p_{0}\right)}
\end{aligned}
$$

Thus

$$
\mathrm{d}\left(p_{t}^{+}, o\right)-\mathrm{d}\left(p_{t}^{-}, o\right) \geq \cos \mathrm{d}\left(p_{t}^{-}, o\right)-\cos \mathrm{d}\left(p_{t}^{+}, o\right)=c \sin \mathrm{d}\left(p_{t}^{+}, p_{0}\right)
$$

where $c=\left(\cos \mathrm{d}\left(p_{1}^{-}, o\right)-\cos \mathrm{d}\left(p_{1}^{+}, o\right)\right) / \sin \mathrm{d}\left(p_{1}^{+}, p_{0}\right)$ is a positive constant depending only on $K \cap \ell$. Since $\rho_{K}\left(\zeta^{+}(t)\right)=\mathrm{d}\left(p_{t}^{+}, o\right)$ and $\rho_{K}\left(\zeta^{-}(t)\right)=$ $\mathrm{d}\left(p_{t}^{-}, o\right)$, we have the strict inequality

$$
\rho_{K}\left(\zeta^{+}(t)\right)>\rho_{K}\left(\zeta^{-}(t)\right), \quad t \in(0,1] .
$$

If $F$ is a strictly increasing function (as in Lemma 5.4), then

$$
\int_{0}^{1} F\left(\rho_{K}\left(\zeta^{+}(t)\right)\right) d t>\int_{0}^{1} F\left(\rho_{K}\left(\zeta^{-}(t)\right)\right) d t
$$


Since $\zeta_{\varepsilon} \rightarrow \zeta$ uniformly as $\varepsilon \rightarrow 0$, the same strict inequality holds for $\zeta_{\varepsilon}$ instead of $\zeta$ for $\varepsilon>0$ small enough. For such small $\varepsilon>0$, apply Lemma 5.4 to complete the proof in this case.

Case (3): Let $\ell^{+}$be the line through $p_{0}, p_{1}$, and $\ell_{\varepsilon}^{+}$be the line that is parallel to $\ell^{+}$but distance $\varepsilon$ farther away from the origin. Similarly, $\ell^{-}$ stands for the line through $q_{0}, q_{1}$, and $\ell_{\delta}^{-}$for the line that is parallel to $\ell^{-}$ but distance $\delta$ closer to the origin.

Let $B$ be the centered ball containing points $p_{0}, p_{1}, q_{0}, q_{1}$ on its boundary. Consider the subregions $B_{\varepsilon}^{+}$and $B_{\delta}^{-}$of $B$ enclosed by $\ell^{+}, \ell_{\varepsilon}^{+}$and by $\ell^{-}, \ell_{\delta}^{-}$. Then $\tilde{K}$ is obtained from $K$ by adding the region $B_{\varepsilon}^{+}$and then removing the region outside the line $\ell_{\delta}^{-}$. It means that $\tilde{K} \backslash K=B_{\varepsilon}^{+} \backslash B_{\delta}^{-}$and $K \backslash \tilde{K}$ is the region enclosed by $\ell^{-}, \ell_{\delta}^{-}, K$. Here, $\delta=\delta(\varepsilon)$ is chosen so that $\operatorname{vol}(\tilde{K} \backslash K)=\operatorname{vol}(K \backslash \tilde{K})$.

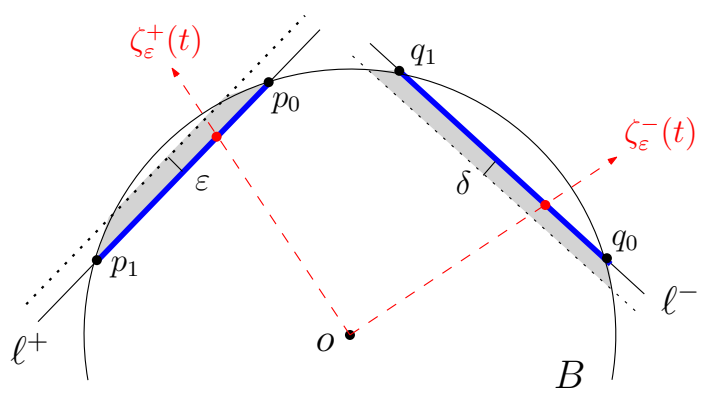

Let $\xi_{\varepsilon}^{+}, \zeta_{\varepsilon}^{+}, \zeta_{\varepsilon}^{-}$be the inverse angular area functions of $B_{\varepsilon}^{+}, \tilde{K} \backslash K, K \backslash \tilde{K}$, respectively. If $\left\{p_{0}, p_{1}\right\} \cap\left\{q_{0}, q_{1}\right\}=\emptyset$, then $\xi_{\varepsilon}^{+}=\zeta_{\varepsilon}^{+}$for small $\varepsilon>0$. Otherwise, it follows from Lemma 5.5 that $\left\|\xi_{\varepsilon}^{+}-\zeta_{\varepsilon}^{+}\right\|^{2}$ is less than a constant multiple of

$$
\frac{\operatorname{vol}\left(B_{\varepsilon}^{+} \Delta(\tilde{K} \backslash K)\right)}{\operatorname{vol}\left(B_{\varepsilon}^{+} \cap(\tilde{K} \backslash K)\right)} \leq \frac{\operatorname{vol}\left(B_{\varepsilon}^{+} \cap B_{\delta}^{-}\right)}{\frac{1}{2} \operatorname{vol}\left(B_{\varepsilon}^{+}\right)} \leq \frac{c_{1} \varepsilon \delta}{c_{2} \varepsilon}=c_{3} \delta,
$$

where the constants $c_{1}, c_{2}, c_{3}$ only depend on $\ell^{+}, \ell^{-}$, and $B$.

Applying Lemma 5.6 to the body $B$ and the line $\ell^{+}$we have the following: as $\varepsilon \rightarrow 0$ the function $\xi_{\varepsilon}^{+}$(and thus $\zeta_{\varepsilon}^{+}$as well) uniformly converges to $\zeta^{+}$ such that for each $t \in[0,1]$ the point $p_{t} \in \ell^{+}$in the direction of $\zeta^{+}(t)$ satisfies

$$
\cos \mathrm{d}\left(p_{t}, p_{*}\right)=(1-t) \cos \mathrm{d}\left(p_{0}, p_{*}\right)+t \cos \mathrm{d}\left(p_{1}, p_{*}\right)
$$

where $p_{*} \in \ell^{+}$lies on the boundary of $\mathbb{S}_{+}^{2}$. Since

$$
\left|\mathrm{d}\left(p_{t}, p_{*}\right)-\mathrm{d}\left(p_{1 / 2}, p_{*}\right)\right|=\mathrm{d}\left(p_{t}, p_{1 / 2}\right)
$$

and

$$
\mathrm{d}\left(p_{1 / 2}, p_{*}\right)=\frac{\mathrm{d}\left(p_{0}, p_{*}\right)+\mathrm{d}\left(p_{1}, p_{*}\right)}{2}=\frac{\pi}{2},
$$


we have

$$
\begin{aligned}
\sin \mathrm{d}\left(p_{t}, p_{1 / 2}\right) & =\sin \left|\mathrm{d}\left(p_{t}, p_{*}\right)-\pi / 2\right|=\left|\cos \mathrm{d}\left(p_{t}, p_{*}\right)\right| \\
& =\left|(1-t) \cos \mathrm{d}\left(p_{0}, p_{*}\right)+t \cos \mathrm{d}\left(p_{1}, p_{*}\right)\right| \\
& =\left|(1-2 t) \cos \mathrm{d}\left(p_{0}, p_{*}\right)\right|=|1-2 t| \sin \mathrm{d}\left(p_{0}, p_{1 / 2}\right) .
\end{aligned}
$$

The (spherical) Pythagorean theorem for the right triangle with vertices $o, p_{1 / 2}, p_{t}$ yields

$$
\cos \mathrm{d}\left(p_{t}, o\right)=\cos \mathrm{d}\left(p_{1 / 2}, o\right) \cos \mathrm{d}\left(p_{t}, p_{1 / 2}\right),
$$

and hence

$$
\frac{\cos \mathrm{d}\left(p_{t}, o\right)}{\cos \mathrm{d}\left(p_{0}, o\right)}=\frac{\cos \mathrm{d}\left(p_{t}, p_{1 / 2}\right)}{\cos \mathrm{d}\left(p_{0}, p_{1 / 2}\right)}=\sqrt{\frac{1-\sin ^{2} \mathrm{~d}\left(p_{t}, p_{1 / 2}\right)}{\cos ^{2} \mathrm{~d}\left(p_{0}, p_{1 / 2}\right)}}
$$

Substituting (15) into the above equation, we get

$$
\cos \mathrm{d}\left(p_{t}, o\right)=\cos \mathrm{d}\left(p_{0}, o\right) \sqrt{1+4 t(1-t) \tan ^{2} \mathrm{~d}\left(p_{0}, p_{1 / 2}\right)} .
$$

Similarly, the function $\zeta_{\varepsilon}^{-}$uniformly converges to $\zeta^{-}$such that for each $t$ the point $q_{t} \in \ell^{-}$in the direction of $\zeta^{-}(t)$ satisfies

$$
\cos \mathrm{d}\left(q_{t}, o\right)=\cos \mathrm{d}\left(q_{0}, o\right) \sqrt{1+4 t(1-t) \tan ^{2} \mathrm{~d}\left(q_{0}, q_{1 / 2}\right)} .
$$

Since $\mathrm{d}\left(p_{0}, o\right)=\mathrm{d}\left(q_{0}, o\right)$ and $\mathrm{d}\left(p_{0}, p_{1 / 2}\right)<\mathrm{d}\left(q_{0}, q_{1 / 2}\right)$, the above two equations imply

$$
\rho_{K}\left(\zeta^{+}(t)\right)=\mathrm{d}\left(p_{t}, o\right)>\mathrm{d}\left(q_{t}, o\right)=\rho_{K}\left(\zeta^{-}(t)\right), \quad t \in(0,1) .
$$

As in Case (3), use the uniform convergence of $\zeta_{\varepsilon}$ to $\zeta$ to get a strict inequality needed for Lemma 5.4 .

Case (4): Suppose that an origin-symmetric convex body $K$ does not contain any type of arcs on its boundary corresponding to Cases (1) and (2). As we will see below this implies that the boundary of $K$ consists of (possibly infinitely many) edges whose endpoints lie on the same centered circle, as well as their limiting points. Consider the set $\mathcal{I}$ of all edges of $K$. Because of Case (2), we can assume that for any $I \in \mathcal{I}$ both endpoints of $I$ are the same distance from the origin, which we will denote by $r(I)$. Let $R=\max _{u \in S^{1}} \rho_{K}(u)$. We claim that there is no edge $I$ with $r(I)<R$. To reach a contradiction, assume that there exists an edge $I_{0}$ with $r\left(I_{0}\right)<R$. We will show that the sets $\mathcal{I}_{\epsilon}=\{I \in \mathcal{I}: R-\varepsilon<r(I)<R\}$ are not empty (and therefore have infinitely many edges) for every $\epsilon>0$. Indeed, suppose that $\mathcal{I}_{\epsilon}$ is empty for some $\epsilon>0$. Consider the following closed subsets of $\partial K: A_{1}=\{x \in \partial K: d(x, o)=R\}$ and $A_{2}=\{x \in \partial K: d(x, o)=R-\epsilon\}$. Let $\gamma \subset \partial K$ be an arc of minimal length connecting $A_{1}$ and $A_{2}$. This arc must contain an edge, since we ruled out Case (1). But this edge cannot have $r(I)<R$, since $\mathcal{I}_{\epsilon}$ is empty and for any interior point $x$ of $\gamma$ we have $d(x, o)>R-\epsilon$. Similarly, $\gamma$ cannot contain an edge with $r(I)=R$ since for any interior point $x$ of $\gamma$ we have $d(x, o)<R$. Contradiction. Thus the 
number of edges in $\mathcal{I}_{\epsilon}$ for any $\varepsilon>0$ should be infinite. But this is also impossible. Just fix $I \in \mathcal{I}$ with $r(I)<R$ and choose $J \in \mathcal{I}$ with $r(I)<r(J)<R$, whose length is small enough to ensure $\mathrm{d}(x, 0)>r(I)$ for all $x \in J$. Use the same argument as in Case (1) for $I, J$ instead of the arcs corresponding to $U, V$; that is, to get $\tilde{K}$ cut off a small piece of $K$ around $I$ by a parallel line and take a convex hull with a point close to $J$. Thus we conclude that $r(I)=R$ for all $I \in \mathcal{I}$. Finally, ruling out the arcs corresponding to Case (3), we see that $K$ is reduced to a regular spherical polygon with $2 m$ edges for some $m \geq 2$.

Let $P$ be the body in $\mathbb{R}^{2}$ with radial function $\rho(x)=\tan \rho_{K}(x)$. Then $P \subset \mathbb{R}^{2}$ is a (Euclidean) regular $2 m$-polygon centered at the origin. If $P$ has an inscribed circle of radius $r$, its radial function $\rho$ can be written as

$$
\rho(x)=r \sec \left(x-\frac{\pi}{2 m}\right), \quad x \in[0, \pi / m]
$$

and extended with period $\frac{\pi}{m}$ to other $x \in[0,2 \pi]$. Define the origin-symmetric body $\bar{P} \subset \mathbb{R}^{2}$ by its radial function $\bar{\rho}$ as follows.

$$
\bar{\rho}(x)= \begin{cases}\rho\left(\frac{x}{2}\right), & 0 \leq x \leq \frac{2 \pi}{m}, \\ \rho(x), & \frac{2 \pi}{m} \leq x \leq \pi, \\ \bar{\rho}(x-\pi), & \pi \leq x \leq 2 \pi .\end{cases}
$$

Then we can use the formula of the curvature of a planar polar curve to show that $\bar{P}$ is still convex and moreover its boundary contains a strictly convex arc. Indeed, the signed curvature of $\partial \bar{P}$ at $x \in\left(0, \frac{2 \pi}{m}\right)$ is

$$
\begin{aligned}
\bar{\kappa}(x) & =\frac{\bar{\rho}^{2}(x)+2\left(\bar{\rho}^{\prime}(x)\right)^{2}-\bar{\rho}(x) \bar{\rho}^{\prime \prime}(x)}{\left(\bar{\rho}^{2}(x)+\left(\bar{\rho}^{\prime}(x)\right)^{2}\right)^{3 / 2}}=\frac{\rho\left(\frac{x}{2}\right)^{2}+\frac{1}{2} \rho^{\prime}\left(\frac{x}{2}\right)^{2}-\frac{1}{4} \rho\left(\frac{x}{2}\right) \rho^{\prime \prime}\left(\frac{x}{2}\right)}{\left(\bar{\rho}^{2}(x)+\left(\bar{\rho}^{\prime}(x)\right)^{2}\right)^{3 / 2}} \\
& =\frac{3}{4} \cdot \frac{\bar{\rho}^{2}(x)}{\left(\bar{\rho}^{2}(x)+\left(\bar{\rho}^{\prime}(x)\right)^{2}\right)^{3 / 2}}+\frac{1}{4} \cdot \frac{\rho\left(\frac{x}{2}\right)^{2}+2 \rho^{\prime}\left(\frac{x}{2}\right)^{2}-\rho\left(\frac{x}{2}\right) \rho^{\prime \prime}\left(\frac{x}{2}\right)}{\left(\bar{\rho}^{2}(x)+\left(\bar{\rho}^{\prime}(x)\right)^{2}\right)^{3 / 2}}
\end{aligned}
$$

Note that $\rho\left(\frac{x}{2}\right)^{2}+2 \rho^{\prime}\left(\frac{x}{2}\right)^{2}-\rho\left(\frac{x}{2}\right) \rho^{\prime \prime}\left(\frac{x}{2}\right)=0$ because the curvature of $\partial P$ at $x / 2 \in(0, \pi / m)$ is equal to 0 . Thus,

$$
\bar{\kappa}(x)=\frac{3 \bar{\rho}^{2}(x)}{4\left(\bar{\rho}^{2}(x)+\left(\bar{\rho}^{\prime}(x)\right)^{2}\right)^{3 / 2}}>0, \quad x \in(0,2 \pi / m) .
$$

This means that the arc of $\bar{P}$ corresponding to $0<x<2 \pi / m$ is strictly convex. To prove convexity of the entire body $\bar{P}$ we need to check what happens at the endpoints of this arc. It suffices to find a supporting line of $\bar{P}$ at each of these endpoints that does not intersect the interior of $\bar{P}$. This is true since $\bar{P}$ is inscribed in the circle of radius $r \sec \left(\frac{\pi}{2 m}\right)$ and those endpoints lie on this circle. 
Thus the body $\tilde{K}$ in $\mathbb{S}_{+}^{2}$ defined by $\tan \rho_{\tilde{K}}(x)=\bar{\rho}(x)$ is an origin-symmetric convex body such that

$$
\begin{aligned}
\operatorname{vol}(\tilde{K}) & =2 \int_{0}^{\frac{2 \pi}{m}}\left[1-\cos \rho_{K}(x / 2)\right] d x+2(m-2) \int_{0}^{\frac{\pi}{m}}\left[1-\cos \rho_{K}(x)\right] d x \\
& =2 m \int_{0}^{\frac{\pi}{m}}\left[1-\cos \rho_{K}(x)\right] d x=\operatorname{vol}(K)
\end{aligned}
$$

and $\int_{0}^{2 \pi} \rho_{\tilde{K}}^{2}(x) d x=\int_{0}^{2 \pi} \rho_{K}^{2}(x) d x$ similarly. Moreover, due to Case (1), $\tilde{K}$ cannot be a maximizer because it contains a strictly convex arc. Thus $K$ is not a maximizer.

To finish the proof it remains to show that

$$
\int_{S^{1}} \operatorname{vol}\left(K \cap \xi^{\perp}\right)^{2} d \xi=16 \int_{0}^{\pi / 2} \arctan ^{2}\left(\frac{\tan (\operatorname{vol}(K) / 4)}{\cos \theta}\right) d \theta
$$

if $K$ is an origin-symmetric lune. This is true because the volume and the radial function of an origin-symmetric lune $K$ can be written as

$$
\operatorname{vol}(K)=4 w, \quad \tan \rho_{K}(\cdot)=\frac{\tan w}{|\langle\cdot, u\rangle|}
$$

where $w \in\left(0, \frac{\pi}{2}\right)$ and $u \in S^{1}$ satisfy $w=\rho_{K}(u)=\min _{x \in K} \rho_{K}(x)$.

\section{Busemann's interseCtion INEQUALity IN $\mathbb{S}_{+}^{n}, n \geq 3$}

In this section we study the maximizing and minimizing problems for the quantity

$$
\int_{S^{n-1}} \operatorname{vol}\left(K \cap \xi^{\perp}\right)^{n} d \xi
$$

in the class of star bodies of a fixed volume in the spherical space $\mathbb{S}_{+}^{n}$ when the dimension $n$ is 3 or higher.

Recall that in the hyperbolic space of any dimension the centered balls are the unique maximizers of this quantity. On the other hand, the centered balls in $\mathbb{S}_{+}^{2}$ are the unique minimizers. Thus it is quite surprising that the balls in $\mathbb{S}_{+}^{n}, n \geq 3$, are neither minimizers nor maximizers (even in the class of origin-symmetric convex bodies).

Theorem 6.1. The centered ball of any radius is neither a minimizer nor a maximizer of the integral $\int_{S^{n-1}} \operatorname{vol}\left(K \cap \xi^{\perp}\right)^{n} d \xi$ in the class of originsymmetric convex bodies $K \subset \mathbb{S}_{+}^{n}, n \geq 3$, of a fixed volume.

Proof. We will show that centered balls cannot even be local minimizers or local maximizers of the integral in question.

Let $B \subset \mathbb{S}_{+}^{n}$ be the centered ball of radius $r \in(0, \pi / 2)$ and $K \subset \mathbb{S}_{+}^{n}$ be an origin-symmetric convex body of the same volume as $B$. Let $f=\rho_{K}-r$ 
and denote $\varepsilon=\|f\|_{L^{\infty}\left(S^{n-1}\right)}$ and $\delta=\|f\|_{L^{2}\left(S^{n-1}\right)}$. We will assume $\varepsilon$, and therefore $\delta$, to be sufficiently small. We have

$$
\operatorname{vol}(K)=\int_{S^{n-1}} \int_{0}^{\rho_{K}(u)} \sin ^{n-1} t d t d u=\int_{S^{n-1}} \phi_{n}\left(\rho_{K}(u)\right) d u
$$

and $\operatorname{vol}(B)=\int_{S^{n-1}} \phi_{n}(r) d u$, where $\phi_{n}(x)=\int_{0}^{x} \sin ^{n-1} t d t$. Using the Taylor expansion of $\phi_{n}$ at $x=r$, we get

$$
\phi_{n}(x)-\phi_{n}(r)=\sin ^{n-1} r\left[(x-r)+\frac{n-1}{2 \tan r}(x-r)^{2}\right]+O\left(|x-r|^{3}\right) .
$$

Putting $\rho_{K}$ instead of $x$ in the above expansion and integrating both sides yield

$$
\begin{aligned}
0 & =\operatorname{vol}(K)-\operatorname{vol}(B)=\int_{S^{n-1}}\left[\phi_{n}\left(\rho_{K}(u)\right)-\phi_{n}(r)\right] d u \\
& =\sin ^{n-1} r\left(\int_{S^{n-1}}\left[f(u)+\frac{n-1}{2 \tan r} f(u)^{2}\right] d u\right)+O\left(\int_{S^{n-1}}|f(u)|^{3} d u\right) .
\end{aligned}
$$

Let $c_{0}=\frac{n-1}{2 \tan r}$. Since

$$
\int_{S^{n-1}}|f(u)|^{3} d u \leq \varepsilon \int_{S^{n-1}}|f(u)|^{2} d u=\varepsilon \delta^{2},
$$

the above equality implies

$$
\int_{S^{n-1}} f(u) d u=-c_{0} \int_{S^{n-1}} f^{2}(u) d u+O\left(\varepsilon \delta^{2}\right)=-c_{0} \delta^{2}+O\left(\varepsilon \delta^{2}\right) .
$$

We now compute the volumes of the sections of $K$. For each $\xi \in S^{n-1}$ we have

$$
\operatorname{vol}\left(K \cap \xi^{\perp}\right)=\int_{S^{n-1} \cap \xi^{\perp}} \phi_{n-1}\left(\rho_{K}(u)\right) d u=R\left(\phi_{n-1} \circ \rho_{K}\right)(\xi) .
$$

Expanding $\phi_{n-1}$ at $r$, we get

$$
\begin{aligned}
\operatorname{vol}\left(K \cap \xi^{\perp}\right) & =R\left[\phi_{n-1}(r)+\sin ^{n-2} r\left(f+\frac{n-2}{2 \tan r} f^{2}\right)+O\left(|f|^{3}\right)\right](\xi) \\
& =\operatorname{vol}\left(B \cap \xi^{\perp}\right)+c_{1} R\left(f+c_{2} f^{2}\right)(\xi)+O\left(R|f|^{3}(\xi)\right),
\end{aligned}
$$

where $c_{1}=\sin ^{n-2} r$ and $c_{2}=\frac{n-2}{2 \tan r}$. 
Raising both sides to the $n$th power, integrating over $S^{n-1}$, and using (16), we have

$$
\begin{aligned}
& \int_{S^{n-1}} \operatorname{vol}\left(K \cap \xi^{\perp}\right)^{n} d \xi-\int_{S^{n-1}} \operatorname{vol}\left(B \cap \xi^{\perp}\right)^{n} d \xi \\
& =\int_{S^{n-1}}\left(c_{3}\left[c_{1} R\left(f+c_{2} f^{2}\right)\right]+c_{4}\left[c_{1} R\left(f+c_{2} f^{2}\right)\right]^{2}\right)(\xi) d \xi+O\left(\varepsilon \delta^{2}\right) \\
& =c_{1} c_{3}\left|S^{n-2}\right| \int_{S^{n-1}}\left(f+c_{2} f^{2}\right)(\xi) d \xi+c_{1}^{2} c_{4} \int_{S^{n-1}}(R f)^{2}(\xi) d \xi+O\left(\varepsilon \delta^{2}\right) \\
& =-c_{1} c_{3}\left(c_{0}-c_{2}\right)\left|S^{n-2}\right| \int_{S^{n-1}} f^{2}(\xi) d \xi+c_{1}^{2} c_{4} \int_{S^{n-1}}(R f)^{2}(\xi) d \xi+O\left(\varepsilon \delta^{2}\right) \\
& =-c_{1}^{2} c_{4} \int_{S^{n-1}}\left[c_{5} f^{2}(\xi)-(R f)^{2}(\xi)\right] d \xi+O\left(\varepsilon \delta^{2}\right),
\end{aligned}
$$

where $c_{3}=n \operatorname{vol}\left(B \cap \xi^{\perp}\right)^{n-1}, c_{4}=\frac{n(n-1)}{2} \operatorname{vol}\left(B \cap \xi^{\perp}\right)^{n-2}$, and

$$
c_{5}=\frac{c_{1} c_{3}\left(c_{0}-c_{2}\right)\left|S^{n-2}\right|}{c_{1}^{2} c_{4}}=\frac{\left|S^{n-2}\right| \operatorname{vol}\left(B \cap \xi^{\perp}\right)}{(n-1) \tan r \sin ^{n-2} r}<\frac{\left|S^{n-2}\right|^{2}}{(n-1)^{2}} .
$$

The above inequality comes from

$$
\frac{\operatorname{vol}\left(B \cap \xi^{\perp}\right)}{\left|S^{n-2}\right|}=\int_{0}^{r} \sin ^{n-2} t d t<\frac{1}{\cos r} \int_{0}^{r} \cos t \sin ^{n-2} t d t=\frac{\tan r \sin ^{n-2} r}{n-1} .
$$

The remainder term in (17) is obtained from the following relations:

$$
\begin{aligned}
\int_{S^{n-1}} R|f|^{3}(\xi) d \xi= & \left|S^{n-2}\right| \int_{S^{n-1}}|f|^{3}(\xi) d \xi=O\left(\varepsilon \delta^{2}\right) \\
\left|\int_{S^{n-1}} R f(\xi) R f^{2}(\xi) d \xi\right| & \leq \varepsilon \int_{S^{n-1}}(R|f|(\xi))^{2} d \xi \\
& \leq \varepsilon\left|S^{n-2}\right|^{2} \int_{S^{n-1}} f^{2}(\xi) d \xi=O\left(\varepsilon \delta^{2}\right)
\end{aligned}
$$

and

$$
\begin{aligned}
& \int_{S^{n-1}}\left|R\left(f+c_{2} f^{2}\right)\right|^{3}(\xi) d \xi \leq\left(1+c_{2} \varepsilon\right)^{3} \int_{S^{n-1}} R|f|^{3}(\xi) d \xi \\
& \leq \varepsilon\left(1+c_{2} \varepsilon\right)^{3}\left|S^{n-2}\right| \int_{S^{n-1}}(R|f|)^{2}(\xi) d \xi \\
& \leq \varepsilon\left(1+c_{2} \varepsilon\right)^{3}\left|S^{n-2}\right|^{3} \int_{S^{n-1}} f^{2}(\xi) d \xi=O\left(\varepsilon \delta^{2}\right),
\end{aligned}
$$

where we used (4) and (5).

To finish the proof, we provide examples of two origin-symmetric convex bodies for which the expression in (17) has opposite signs. Let

$$
f=\alpha+\beta H_{k},
$$

where $H_{k}$ is a spherical harmonic of an even degree $k>0$ with $\left\|H_{k}\right\|_{L^{2}\left(S^{n-1}\right)}=$ 1 , and $\alpha, \beta$ are the numbers satisfying the conditions $\delta=\|f\|_{L^{2}\left(S^{n-1}\right)}$ and 
$\operatorname{vol}(K)=\operatorname{vol}(B)$. The first condition implies $\left|S^{n-1}\right| \alpha^{2}+\beta^{2}=\delta^{2}$, and the second one gives

$$
\alpha=\frac{1}{\left|S^{n-1}\right|} \int_{S^{n-1}} f(\xi) d \xi=-\frac{c_{0} \delta^{2}}{\left|S^{n-1}\right|}+O\left(\varepsilon \delta^{2}\right),
$$

which follows from condition (16). Note that $\alpha \rightarrow 0$ and $\beta \rightarrow 0$ as $\delta \rightarrow 0$. Choosing $\delta$ sufficiently small, we can guarantee that the body $K$ is convex.

Now compute the sign of (17) for such a body $K$. Since $R H_{k}=\lambda_{k} H_{k}$, $\int_{S^{n-1}} H_{k}=0$ and $\alpha=O\left(\delta^{2}\right)$, we have

$$
\begin{aligned}
& \int_{S^{n-1}}\left[c_{5} f^{2}(\xi)-(R f)^{2}(\xi)\right] d \xi \\
& =\int_{S^{n-1}}\left[c_{5}\left(\alpha^{2}+\beta^{2} H_{k}^{2}(\xi)\right)-\left(\lambda_{0}^{2} \alpha^{2}+\beta^{2} \lambda_{k}^{2} H_{k}^{2}(\xi)\right)\right] d \xi \\
& =\left(c_{5}-\lambda_{k}^{2}\right) \int_{S^{n-1}}\left[\alpha^{2}+\beta^{2} H_{k}^{2}(\xi)\right] d \xi+\left(\lambda_{k}^{2}-\lambda_{0}^{2}\right)\left|S^{n-1}\right| \alpha^{2} \\
& =\left(c_{5}-\lambda_{k}^{2}\right) \delta^{2}+O\left(\delta^{4}\right) .
\end{aligned}
$$

Thus

$$
17)=-c_{1}^{2} c_{4}\left(c_{5}-\lambda_{k}^{2}\right) \delta^{2}+O\left(\varepsilon \delta^{2}\right) .
$$

Choosing $k$ large enough, so that $\lambda_{k}^{2}<c_{5}$ and $\delta>0$ (and therefore $\epsilon$ ) small enough, we can guarantee that the sign of (17) is negative, which means

$$
\int_{S^{n-1}} \operatorname{vol}\left(K \cap \xi^{\perp}\right)^{n} d \xi<\int_{S^{n-1}} \operatorname{vol}\left(B \cap \xi^{\perp}\right)^{n} d \xi
$$

On the other hand, if $k=2$ then $\lambda_{2}^{2}=\left|S^{n-2}\right|^{2} /(n-1)^{2}>c_{5}$, which makes the sign of (17) positive and yields the reversed inequality:

$$
\int_{S^{n-1}} \operatorname{vol}\left(K \cap \xi^{\perp}\right)^{n} d \xi>\int_{S^{n-1}} \operatorname{vol}\left(B \cap \xi^{\perp}\right)^{n} d \xi
$$

Next we will solve the minimizing problem in the class of star bodies, not necessarily origin-symmetric. For this we need the following lemma. We will use it to show that the constant in Theorem 6.3 is optimal.

Lemma 6.2. Let $C$ be a spherical cap in $S^{n-1}, n \geq 3$, of the form

$$
C=\left\{x \in S^{n-1}:\langle x, u\rangle \geq \alpha\right\}, \quad u \in S^{n-1}, \quad 0<\alpha<1 .
$$

Then, for each $\lambda \in(0,1)$ and small $\varepsilon>0$, there exists a subset $A$ of $C$ such that $|A|=\lambda|C|$ and for each $\xi \in S^{n-1}$

$$
\left|A \cap \xi^{\perp}\right| \leq \lambda\left|C \cap \xi^{\perp}\right|+\varepsilon .
$$

Proof. Let $\delta>0$ be small and $0<\gamma<1$, both of which will be fixed later according to $\varepsilon>0$. Write $C$ as the following union of disjoint narrow strips.

$$
C=\left(A_{1} \cup A_{2} \cup \cdots \cup A_{N}\right) \cup\left(B_{1} \cup B_{2} \cup \cdots \cup B_{N}\right)
$$


where $N$ is the smallest integer greater than $(1-\alpha) / \delta$ and, for each $k=$ $1, \ldots, N$,

$$
\begin{aligned}
& A_{k}=\{x \in C: \alpha+(k-\gamma) \delta \leq\langle x, u\rangle \leq \alpha+k \delta\} \\
& B_{k}=\{x \in C: \alpha+(k-1) \delta<\langle x, u\rangle<\alpha+(k-\gamma) \delta\} .
\end{aligned}
$$

Note that $A_{N}$ may be empty or have a smaller height than the rest of $A_{k}$. $B_{N}$ cannot be empty, but it may possibly have a smaller height than the rest of $B_{k}$.

Then we have

$$
\left|A_{k}\right|=\int_{\alpha+(k-\gamma) \delta}^{\alpha+k \delta} f_{n}(t) d t \quad \text { and } \quad\left|B_{k}\right|=\int_{\alpha+(k-1) \delta}^{\alpha+(k-\gamma) \delta} f_{n}(t) d t
$$

where $f_{n}(t)=\left|S^{n-2}\right|\left(1-t^{2}\right)^{(n-3) / 2}$ if $0 \leq t \leq 1$ and $f_{n}(t)=0$ if $t>1$. (This extension of the function $f_{n}$ to the values $t>1$ is dictated by the possibility that $\alpha+N \delta>1$ ).

Since $f_{n}$ is non-increasing, we have

$$
\begin{aligned}
\gamma \delta \cdot f_{n}(\alpha+(k-\gamma) \delta) & \geq\left|A_{k}\right| \geq \gamma \delta \cdot f_{n}(\alpha+k \delta) \\
(1-\gamma) \delta \cdot f_{n}(\alpha+(k-1) \delta) & \geq\left|B_{k}\right| \geq(1-\gamma) \delta \cdot f_{n}(\alpha+(k-\gamma) \delta) .
\end{aligned}
$$

Assuming that $B_{N+1}=\emptyset$, this implies

$$
\frac{\left|B_{k}\right|}{1-\gamma} \geq \frac{\left|A_{k}\right|}{\gamma} \geq \frac{\left|B_{k+1}\right|}{1-\gamma}, \quad k=1,2, \ldots, N .
$$

Let $A=A_{1} \cup A_{2} \cup \cdots \cup A_{N}$. Using the left inequality of (18), we get

$$
\begin{aligned}
|A| & =\sum_{k=1}^{N}\left|A_{k}\right|=\sum_{k=1}^{N}\left((1-\gamma)\left|A_{k}\right|+\gamma\left|A_{k}\right|\right) \\
& \leq \sum_{k=1}^{N}\left(\gamma\left|B_{k}\right|+\gamma\left|A_{k}\right|\right)=\gamma|C| .
\end{aligned}
$$

Also, using the right inequality of (18), we get

$$
\begin{aligned}
|A| & =\sum_{k=1}^{N}\left|A_{k}\right|=\sum_{k=1}^{N}\left((1-\gamma)\left|A_{k}\right|+\gamma\left|A_{k}\right|\right) \\
& \geq \sum_{k=1}^{N}\left(\gamma\left|B_{k+1}\right|+\gamma\left|A_{k}\right|\right)=\gamma\left(|C|-\left|B_{1}\right|\right) .
\end{aligned}
$$

Note that $|A|$ increases from 0 to $|C|$ as $\gamma$ changes from 0 to 1 , so we can fix $\gamma=\gamma(\delta)$ with $|A|=\lambda|C|$. Then (19), (20) imply that $\lambda \leq \gamma$ and

$$
\gamma \leq \frac{\lambda|C|}{|C|-\left|B_{1}\right|} \leq \frac{\lambda|C|}{|C|-\delta f_{n}(\alpha)}=\frac{\lambda}{1-\delta f_{n}(\alpha) /|C|} .
$$


Assuming that $\delta<f_{n}(\alpha) /(2|C|)$, we get

$$
\gamma \leq \lambda\left(1+\frac{2 f_{n}(\alpha)}{|C|} \delta\right)=\lambda+c_{1} \delta
$$

where $c_{1}=2 \lambda f_{n}(\alpha) /|C|$.

To check the statement for $A \cap \xi^{\perp}$, we write $\xi \in S^{n-1}$ as

$$
\xi=u \sqrt{1-s^{2}}+s v, \quad 0 \leq s \leq 1, \quad v \in S^{n-1} \cap u^{\perp} .
$$

If $0 \leq s<\alpha$, then $C \cap \xi^{\perp}$ is empty; so assume $s \geq \alpha$. Note that for $x \in \xi^{\perp}$,

$$
\langle x, u\rangle=\langle x, u-\langle u, \xi\rangle \xi\rangle=s\langle x, \tilde{u}\rangle,
$$

where

$$
\tilde{u}=\frac{u-\langle u, \xi\rangle \xi}{|u-\langle u, \xi\rangle \xi|}=\frac{u-\langle u, \xi\rangle \xi}{\sqrt{1-\langle u, \xi\rangle^{2}}}=\frac{u-\langle u, \xi\rangle \xi}{s} .
$$

Let $\tilde{\delta}=\delta / s, \tilde{\alpha}=\alpha / s$, and $\tilde{N}$ be the largest integer $k$ such that $A_{k} \cap \xi^{\perp}$ is non-empty. (Note that the definition of $\tilde{N}$ is slightly different from that of $N$ ). If $A_{k} \cap \xi^{\perp}$ is empty for all $k$, then we are done. So we will assume $\tilde{N} \geq 1$. We have

$$
\begin{aligned}
A_{k} \cap \xi^{\perp} & =\left\{x \in C \cap \xi^{\perp}: \alpha+(k-\gamma) \delta \leq\langle x, u\rangle \leq \alpha+k \delta\right\} \\
& =\left\{x \in C \cap \xi^{\perp}: \tilde{\alpha}+(k-\gamma) \tilde{\delta} \leq\langle x, \tilde{u}\rangle \leq \tilde{\alpha}+k \tilde{\delta}\right\}
\end{aligned}
$$

and, similarly,

$$
B_{k} \cap \xi^{\perp}=\left\{x \in C \cap \xi^{\perp}: \tilde{\alpha}+(k-1) \tilde{\delta}<\langle x, \tilde{u}\rangle<\tilde{\alpha}+(k-\gamma) \tilde{\delta}\right\} .
$$

In the case of $n \geq 4$, using the same argument as in 18 for $A \cap \xi^{\perp}, C \cap \xi^{\perp}$, $\tilde{\alpha}, \tilde{\delta}, f_{n-1}$ instead of $A, C, \alpha, \delta, f_{n}$, we have

$$
\frac{\left|B_{k} \cap \xi^{\perp}\right|}{1-\gamma} \geq \frac{\left|A_{k} \cap \xi^{\perp}\right|}{\gamma}, \quad k=1, \ldots, \tilde{N},
$$

since the function $f_{n-1}$ is still non-increasing when $n \geq 4$. So

$$
\left|A \cap \xi^{\perp}\right| \leq \sum_{k=1}^{\tilde{N}}\left(\gamma\left|B_{k} \cap \xi^{\perp}\right|+\gamma\left|A_{k} \cap \xi^{\perp}\right|\right) \leq \gamma\left|C \cap \xi^{\perp}\right| .
$$

Using (21) to bound $\gamma$ and letting $c_{2}=c_{1}\left|S^{n-2}\right|$ yield

$$
\left|A \cap \xi^{\perp}\right| \leq \lambda\left|C \cap \xi^{\perp}\right|+c_{2} \delta .
$$

On the other hand, if $n=3$, the function $f_{n-1}(t)=2 / \sqrt{1-t^{2}}$ is increasing on $(0,1)$, which implies

and hence

$$
\frac{\left|A_{k} \cap \xi^{\perp}\right|}{\gamma} \leq \frac{\left|B_{k+1} \cap \xi^{\perp}\right|}{1-\gamma}, \quad k=1, \ldots, \tilde{N}-1,
$$

$$
\sum_{k=1}^{\tilde{N}-1}\left|A_{k} \cap \xi^{\perp}\right| \leq \sum_{k=1}^{\tilde{N}-1}\left(\gamma\left|B_{k+1} \cap \xi^{\perp}\right|+\gamma\left|A_{k} \cap \xi^{\perp}\right|\right) \leq \gamma\left|C \cap \xi^{\perp}\right| .
$$


Thus

$$
\begin{aligned}
\left|A \cap \xi^{\perp}\right| & \leq \gamma\left|C \cap \xi^{\perp}\right|+\left|A_{\tilde{N}} \cap \xi^{\perp}\right| \leq \gamma\left|C \cap \xi^{\perp}\right|+\int_{1-\tilde{\delta}}^{1} \frac{2 d t}{\sqrt{1-t^{2}}} \\
& \leq \lambda\left|C \cap \xi^{\perp}\right|+c_{2} \delta+2 \arccos (1-\delta / \alpha),
\end{aligned}
$$

where we assumed $\delta<\alpha$.

Finally, choosing an appropriate $\delta>0$, satisfying $c_{2} \delta+2 \arccos (1-\delta / \alpha) \leq$ $\varepsilon$ if $n=3$ and $c_{2} \delta \leq \varepsilon$ otherwise, yields the desired inequality for $\left|A \cap \xi^{\perp}\right|$.

Theorem 6.3. Let $K$ be star-shaped in $\mathbb{S}_{+}^{n}, n \geq 3$. Then

$$
\int_{S^{n-1}} \operatorname{vol}\left(K \cap \xi^{\perp}\right)^{n} d \xi \geq c_{n} \operatorname{vol}(K)^{n}
$$

where $c_{n}=2 \Gamma\left(\frac{n+1}{2}\right)^{n} \Gamma\left(\frac{n}{2}\right)^{-n-1}$ is optimal for any fixed volume.

Proof. We will start with the following inequality

$$
\frac{\phi_{n-1}\left(\frac{\pi}{2}\right)}{\phi_{n}\left(\frac{\pi}{2}\right)} \phi_{n}(x) \leq \phi_{n-1}(x), \quad 0 \leq x \leq \frac{\pi}{2},
$$

where, as before, $\phi_{n}(x)=\int_{0}^{x} \sin ^{n-1} t d t$.

To prove it, consider the function

$$
f(x)=\frac{\phi_{n-1}\left(\frac{\pi}{2}\right)}{\phi_{n}\left(\frac{\pi}{2}\right)} \phi_{n}(x)-\phi_{n-1}(x) .
$$

One can see that $f(0)=f(\pi / 2)=0$. Moreover, $f^{\prime}$ changes its sign exactly once in the interval $(0, \pi / 2)$ and $f^{\prime}(x)>0$ when $x$ is near $\pi / 2$. Thus $f$ is first decreasing and then increasing, which means that $f(x)<0$ when $x \in(0, \pi / 2)$.

Next, using Hölder's inequality, formula (5), and inequality (22), we get

$$
\begin{aligned}
\int_{S^{n-1}} & \operatorname{vol}\left(K \cap \xi^{\perp}\right)^{n} d \xi \\
& =\int_{S^{n-1}}\left(\int_{S^{n-1} \cap \xi^{\perp}} \int_{0}^{\rho_{K}(\theta)} \sin ^{n-2} r d r d \theta\right)^{n} d \xi \\
& \geq\left|S^{n-1}\right|^{-n+1}\left(\int_{S^{n-1}} \int_{S^{n-1} \cap \xi^{\perp}} \phi_{n-1}\left(\rho_{K}(\theta)\right) d \theta d \xi\right)^{n} \\
& =\left|S^{n-1}\right|^{-n+1}\left|S^{n-2}\right|^{n}\left(\int_{S^{n-1}} \phi_{n-1}\left(\rho_{K}(\xi)\right) d \xi\right)^{n} \\
& \geq\left|S^{n-1}\right|^{-n+1}\left|S^{n-2}\right|^{n}\left[\frac{\phi_{n-1}(\pi / 2)}{\phi_{n}(\pi / 2)}\right]^{n}\left(\int_{S^{n-1}} \phi_{n}\left(\rho_{K}(\xi)\right) d \xi\right)^{n} \\
& =c_{n} \operatorname{vol}(K)^{n},
\end{aligned}
$$


where

$$
c_{n}=\left[\frac{\phi_{n-1}(\pi / 2)}{\phi_{n}(\pi / 2)}\right]^{n} \frac{\left|S^{n-2}\right|^{n}}{\left|S^{n-1}\right|^{n-1}}=\frac{\left|S^{n-1}\right|^{n+1}}{\left|S^{n}\right|^{n}}=\frac{2 \Gamma\left(\frac{n+1}{2}\right)^{n}}{\Gamma\left(\frac{n}{2}\right)^{n+1}} .
$$

To show that the constant $c_{n}$ is optimal for any fixed volume we construct a family of origin-symmetric star-shaped sets of a given volume in $\mathbb{S}_{+}^{n}$, for which the infimum of the quantity $\operatorname{vol}(K)^{-n} \int_{S^{n-1}} \operatorname{vol}\left(K \cap \xi^{\perp}\right)^{n}$ is equal to $c_{n}$. Let $0<t<1$ be fixed and take a small $\varepsilon>0$. Consider a spherical cap $C_{\alpha}=\left\{x \in S^{n-1}:\left\langle x, e_{1}\right\rangle \geq \alpha\right\}$ for small $\alpha>0$ with $\left|C_{\alpha}\right|>\frac{t}{2}\left|S^{n-1}\right|$. Applying Lemma 6.2 to $C_{\alpha}$ and $\lambda=\frac{t\left|S^{n-1}\right|}{2\left|C_{\alpha}\right|}$, we get $A_{t} \subset C_{\alpha}$ such that $\left|A_{t}\right|=\lambda\left|C_{\alpha}\right|$ and $\left|A_{t} \cap \xi^{\perp}\right| \leq \lambda\left|C_{\alpha} \cap \xi^{\perp}\right|+\varepsilon$ for each $\xi \in S^{n-1}$. Let $K_{t}$ be the (origin-symmetric) cone with base $A_{t} \cup\left(-A_{t}\right)$. Then

$$
\operatorname{vol}\left(K_{t}\right)=2 \phi_{n}(\pi / 2)\left|A_{t}\right|=2 \lambda \phi_{n}(\pi / 2)\left|C_{\alpha}\right|=t \operatorname{vol}\left(\mathbb{S}_{+}^{n}\right)
$$

and

$$
\begin{array}{r}
\frac{1}{\operatorname{vol}\left(K_{t}\right)^{n}} \int_{S^{n-1}} \operatorname{vol}\left(K_{t} \cap \xi^{\perp}\right)^{n} d \xi=\int_{S^{n-1}}\left(\frac{2 \phi_{n-1}\left(\frac{\pi}{2}\right)\left|A_{t} \cap \xi^{\perp}\right|}{2 \lambda \phi_{n}\left(\frac{\pi}{2}\right)\left|C_{\alpha}\right|}\right)^{n} d \xi \\
\leq \frac{\phi_{n-1}^{n}\left(\frac{\pi}{2}\right)}{\phi_{n}^{n}\left(\frac{\pi}{2}\right)} \int_{S^{n-1}}\left(\frac{\lambda\left|C_{\alpha} \cap \xi^{\perp}\right|+\varepsilon}{\lambda\left|C_{\alpha}\right|}\right)^{n} d \xi .
\end{array}
$$

As $\alpha \rightarrow 0$ and $\varepsilon \rightarrow 0$, the right hand side of the above inequality approaches the constant $c_{n}$.

Remark 6.4. Even though the constant $c_{n}$ in Theorem 6.3 is optimal for star-shaped sets of any fixed volume, the inequality in Theorem 6.3 is strict for most of them. Equality occurs only in special cases, namely, if and only if $K$ is a cone whose base $B \subset S^{n-1}$ is one of the following types:

(1) $B$ or its complement in $S^{n-1}$ is of measure zero.

(2) The intersection of $B$ and the reflection of its complement with respect to the origin is of measure zero.

Proof. Equality in (22) holds only when $x=0$ or $x=\pi / 2$. Thus, $\rho_{K}$ can only take two values: 0 and $\pi / 2$. Furthermore, equality in Hölder's inequality occurs when $\int_{S^{n-1} \cap \xi^{\perp}} \phi_{n-1}\left(\rho_{K}(\theta)\right) d \theta$ is a constant for almost all $\xi \in S^{n-1}$. This means that $\phi_{n-1}\left(\rho_{K}(\theta)\right)+\phi_{n-1}\left(\rho_{K}(-\theta)\right)$ is also a constant for almost all $\theta \in S^{n-1}$. The value of this constant can only be $0, \phi_{n-1}(\pi / 2)$, $2 \phi_{n-1}(\pi / 2)$. Clearly, in the first case we get $\rho_{K}(\theta)=0$, in the second case $\rho_{K}(\theta)=0$ if and only if $\rho_{K}(-\theta)=\pi / 2$, in the third case $\rho_{K}(\theta)=\pi / 2$ (up to sets of measure zero).

Using Lemma 6.2 we can also show that the infimum of $\int_{S^{n-1}}\left|K \cap \xi^{\perp}\right|^{n} d \xi$ in the class of (origin-symmetric) star bodies in $\mathbb{R}^{n}$ or $\mathbb{H}^{n}$ of any fixed volume is zero. 
Theorem 6.5. Let $\mathbb{M}^{n}=\mathbb{R}^{n}$ or $\mathbb{H}^{n}$ for $n \geq 3$ and $\alpha>0$. For each $\eta>0$ there is an origin-symmetric star body $K \subset \mathbb{M}^{n}$ of volume $\alpha$ such that

$$
\int_{S^{n-1}} \operatorname{vol}\left(K \cap \xi^{\perp}\right)^{n} d \xi \leq \eta
$$

Proof. Let $\phi_{n}(x)=\int_{0}^{x} s(t)^{n-1} d t$, where $s(t)=t$ if $\mathbb{M}^{n}=\mathbb{R}^{n}$, and $s(t)=$ $\sinh t$ if $\mathbb{M}^{n}=\mathbb{H}^{n}$. In either case we have

$$
\lim _{x \rightarrow \infty} \phi_{n}(x)=\infty, \quad \lim _{x \rightarrow \infty} \frac{\phi_{n-1}(x)}{\phi_{n}(x)}=\lim _{x \rightarrow \infty} \frac{\phi_{n-1}^{\prime}(x)}{\phi_{n}^{\prime}(x)}=0 .
$$

Fix a spherical cap $C \subset S^{n-1}$ of measure less than $\frac{1}{2}\left|S^{n-1}\right|$. Let $r$ be large enough so that $\phi_{n}(r)>\frac{\alpha}{2|C|}$. Applying Lemma 6.2 for the spherical cap $C$, $\lambda=\frac{\alpha}{2 \phi_{n}(r)|C|}$, and $\varepsilon=\frac{1}{\phi_{n}(r)}$, we can construct a set $A \subset S^{n-1}$ such that $|A|=\lambda|C|$ and for each $\xi \in S^{n-1}$

$$
\left|A \cap \xi^{\perp}\right| \leq \lambda\left|C \cap \xi^{\perp}\right|+\varepsilon=\frac{1}{\phi_{n}(r)} \cdot \frac{\alpha\left|C \cap \xi^{\perp}\right|+2|C|}{2|C|} .
$$

Define the origin-symmetric star-shaped set $K \subset \mathbb{M}^{n}$ by

$$
\rho_{K}(x)= \begin{cases}r, & \text { if } x \in A \cup(-A), \\ 0, & \text { otherwise. }\end{cases}
$$

Then

$$
\operatorname{vol}(K)=\int_{S^{n-1}} \phi_{n}\left(\rho_{K}(x)\right) d x=2 \phi_{n}(r)|A|=2 \phi_{n}(r) \lambda|C|=\alpha
$$

and

$$
\begin{aligned}
\int_{S^{n-1}} \operatorname{vol}\left(K \cap \xi^{\perp}\right)^{n} d \xi & =\int_{S^{n-1}}\left(2 \phi_{n-1}(r)\left|A \cap \xi^{\perp}\right|\right)^{n} d \xi \\
& \leq\left(\frac{\phi_{n-1}(r)}{\phi_{n}(r)}\right)^{n} \int_{S^{n-1}}\left(\frac{\alpha\left|C \cap \xi^{\perp}\right|+2|C|}{|C|}\right)^{n} d \xi
\end{aligned}
$$

Since the integral in the right-hand side is a constant and $\frac{\phi_{n-1}(r)}{\phi_{n}(r)} \rightarrow 0$ as $r \rightarrow \infty$, choosing $r$ large enough, so that the right hand side is less than $\eta$, yields the desired inequality. The statement is now proved for star-shaped sets. By an approximation the statement extends to star bodies.

Question. Is it true that the maximizers in the class of origin-symmetric convex bodies in $\mathbb{S}_{+}^{n}, n \geq 3$, of a fixed volume are the lunes?

\section{Busemann's intersection inequality in a General MEASURE SPACE}

In what follows, $\mu$ will be a measure on $\mathbb{M}^{n}=\mathbb{R}^{n}$ or $\mathbb{H}^{n}$ with density $f$. If $K$ is a star body in $\mathbb{M}^{n}$, then

$$
\mu(K)=\int_{K} f(x) d \operatorname{vol}(x)
$$


and

$$
\mu\left(K \cap \xi^{\perp}\right)=\int_{K \cap \xi^{\perp}} f(x) d \operatorname{vol}(x),
$$

where $d$ vol is the volume element on $\mathbb{M}^{n}$ or the submanifold $\xi^{\perp}$.

Theorem 7.1. Let $K$ be a star body in $\mathbb{M}^{n}=\mathbb{R}^{n}$ or $\mathbb{H}^{n}$ and $\mu$ be a measure on $\mathbb{M}^{n}$, whose density $f$ is radially symmetric and decreasing. Then

$$
\int_{S^{n-1}} \mu\left(K \cap \xi^{\perp}\right)^{n} d \sigma_{n-1}(\xi) \leq[\Psi(\mu(K))]^{n-1}
$$

with equality if and only if $K$ is a ball centered at the origin. Here, $d \sigma_{n-1}$ is the spherical volume element normalized so that $\sigma_{n-1}\left(S^{n-1}\right)=1$, and $\Psi=\psi_{n-1}^{\frac{n}{n-1}} \circ \psi_{n}^{-1}$ is a positive increasing concave function on $[0, \infty)$, where $\psi_{n}(x)$ denotes the measure (with respect to $\mu$ ) of the centered ball in $\mathbb{M}^{n}$ of radius $x \geq 0$.

Proof. The function $\psi_{n}$ can be written as

$$
\psi_{n}(x)=\left|S^{n-1}\right| \int_{0}^{x} f(t) s(t)^{n-1} d t
$$

where $f$ is decreasing on $[0, \infty), s(t)=t$ if $\mathbb{M}^{n}=\mathbb{R}^{n}$ and $s(t)=\sinh t$ if $\mathbb{M}^{n}=\mathbb{H}^{n}$. Note that in either case $s(t)$ is a convex function on $[0, \infty)$. First we show that $\Psi$ is concave. Differentiating $\Psi\left(\psi_{n}(x)\right)=\left[\psi_{n-1}(x)\right]^{\frac{n}{n-1}}$ we have

$$
\Psi^{\prime}\left(\psi_{n}(x)\right)=\frac{\left|S^{n-2}\right|}{\left|S^{n-1}\right|} \frac{n}{n-1}\left[\psi_{n-1}(x)\right]^{\frac{1}{n-1}} / s(x)
$$

and

$\Psi^{\prime \prime}\left(\psi_{n}(x)\right)=\frac{n\left|S^{n-2}\right|\left[\psi_{n-1}(x)\right]^{(2-n) /(n-1)}}{(n-1)\left|S^{n-1}\right|^{2} f(x) s(x)^{n+1}}\left[\frac{\left|S^{n-2}\right| f(x) s(x)^{n-1}}{n-1}-\psi_{n-1}(x) s^{\prime}(x)\right]$.

Since $f$ is decreasing and $s$ is convex, we have

$$
\begin{aligned}
\psi_{n-1}(x) s^{\prime}(x) & =s^{\prime}(x)\left|S^{n-2}\right| \int_{0}^{x} f(t) s(t)^{n-2} d t \\
& >f(x)\left|S^{n-2}\right| \int_{0}^{x} s(t)^{n-2} s^{\prime}(t) d t=\frac{\left|S^{n-2}\right| f(x) s(x)^{n-1}}{n-1} .
\end{aligned}
$$

This implies $\Psi^{\prime \prime}<0$, so $\Psi=\psi_{n-1}^{\frac{n}{n-1}} \circ \psi_{n}^{-1}$ is concave on $(0, \infty)$.

Let $L$ be the star body in $\mathbb{R}^{n}$ with the radial function

$$
\rho_{L}(u)=\left[\frac{\psi_{n-1}\left(\rho_{K}(u)\right)}{\left|B_{2}^{n-1}\right|}\right]^{\frac{1}{n-1}}, \quad u \in S^{n-1} .
$$


Then, for each $\xi \in S^{n-1}$,

$$
\begin{aligned}
\mu\left(K \cap \xi^{\perp}\right) & =\int_{S^{n-1} \cap \xi^{\perp}} \int_{0}^{\rho_{K}(u)} f(t) s(t)^{n-2} d t d u \\
& =\frac{1}{\left|S^{n-2}\right|} \int_{S^{n-1} \cap \xi^{\perp}} \psi_{n-1}\left(\rho_{K}(u)\right) d u \\
& =\frac{1}{n-1} \int_{S^{n-1} \cap \xi^{\perp}} \rho_{L}^{n-1}(u) d u=\left|L \cap \xi^{\perp}\right|^{n} .
\end{aligned}
$$

Busemann's intersection inequality for $L \subset \mathbb{R}^{n}$ implies

$$
\int_{S^{n-1}}\left|L \cap \xi^{\perp}\right|^{n} d \sigma_{n-1}(\xi) \leq c_{1}|L|^{n-1},
$$

where $c_{1}=\left|B_{2}^{n-1}\right|^{n} /\left|B_{2}^{n}\right|^{n-1}$. The concavity of $\Psi$ implies

$$
\begin{aligned}
|L| & =\frac{1}{n} \int_{S^{n-1}} \rho_{L}^{n}(u) d u=\frac{1}{n} \int_{S^{n-1}}\left[\frac{\psi_{n-1}\left(\rho_{K}(u)\right)}{\left|B_{2}^{n-1}\right|}\right]^{\frac{n}{n-1}} d u \\
& =c_{2} \int_{S^{n-1}}\left(\psi_{n-1}^{\frac{n}{n-1}} \circ \psi_{n}^{-1}\right)\left(\psi_{n}\left(\rho_{K}(u)\right)\right) d \sigma_{n-1}(u) \\
& \leq c_{2} \Psi\left(\int_{S^{n-1}} \psi_{n}\left(\rho_{K}(u)\right) d \sigma_{n-1}(u)\right)=c_{2} \Psi(\mu(K)),
\end{aligned}
$$

with $c_{2}=\left|B_{2}^{n}\right| /\left|B_{2}^{n-1}\right|^{n /(n-1)}$. Therefore

$$
\int_{S^{n-1}} \mu\left(K \cap \xi^{\perp}\right)^{n} d \sigma_{n-1}(\xi) \leq c_{1}\left[c_{2} \Psi(\mu(K))\right]^{n-1}=[\Psi(\mu(K))]^{n-1} .
$$

Moreover, the equality case in inequalities (23) and (24) holds if and only if $K$ is a centered ball (we use that $\Psi$ is strictly concave).

Remark 7.2. An important particular case of the previous theorem is the case when the measure $\mu$ is the standard Gaussian measure, i.e.,

$$
\begin{gathered}
\mu(K)=(2 \pi)^{-n / 2} \int_{K} e^{-|x|^{2} / 2} d x \\
\mu\left(K \cap \xi^{\perp}\right)=(2 \pi)^{(-n+1) / 2} \int_{K \cap \xi^{\perp}} e^{-|x|^{2} / 2} d x .
\end{gathered}
$$

Note that one has to adjust the coefficient in Theorem 7.1 since the densities on $\mathbb{R}^{n}$ and $\mathbb{R}^{n-1}$ differ by a factor of $\sqrt{2 \pi}$.

\section{REFERENCES}

[1] H. Busemann, Volume in terms of concurrent cross-sections, Pacific J. Math. 3 (1953), 1-12.

[2] S. Dann, G. Paouris, P. Pivovarov, Bounding marginal densities via affine isoperimetry, Proc. Lond. Math. Soc. 113 (2016), 140-162.

[3] R. J. Gardner, Geometric Tomography. Second edition. Encyclopedia of Mathematics and its Applications, 58. Cambridge University Press, Cambridge, 2006. 
[4] R. J. Gardner, The dual BrunnMinkowski theory for bounded Borel sets: Dual affine quermassintegrals and inequalities, Adv. Math. 216 (2007), 358-386.

[5] E. GRINBERG, Isoperimetric inequalities and identities for $k$-dimensional crosssections of convex bodies, Math. Ann. 291 (1991), 75-86.

[6] H. Groemer, Geometric applications of Fourier series and spherical harmonics. Encyclopedia of Mathematics and its Applications, 61. Cambridge University Press, Cambridge, 1996.

[7] E. Lutwak, Selected affine isoperimetric inequalities, P. M. Gruber, J. M. Wills (Eds.), Handbook of Convex Geometry, North-Holland, Amsterdam (1993), pp. 151-176.

[8] V. YASKIn, The Busemann-Petty problem in hyperbolic and spherical spaces, Adv. Math. 203 (2006), no. 2, 537-553.

Vienna University of Technology, Wiedner Hauptstrasse 8-10, 1040 Vienna, Austria

E-mail address: susanna.dann@tuwien.ac.at

Department of Mathematical and Statistical Sciences, University of AlBerta, Edmonton, Alberta T6G 2G1, Canada

E-mail address: jaegil@ualberta.ca

Department of Mathematical and Statistical Sciences, University of AlBerta, Edmonton, Alberta T6G 2G1, Canada

E-mail address: vladyaskin@math.ualberta.ca 